\title{
An Alternating Sheared AA Pair and Elements of Stability for a Single Sheared Purine-Purine Pair Flanked by Sheared GA Pairs
}

\author{
Supporting Information \\ Gang Chen, Scott D. Kennedy, Jing Qiao, Thomas R. Krugh, and Douglas H. Turner
}

Table S1. List of Distance Restraints (in $\AA$ ) Used for the Structural Modeling of ${ }_{\text {PCCA }}^{\text {GGAG GGCU }}$. See text for Dihedral Angle Restraints. Values in fifth column are measured distances of corresponding internal loop in the crystal structure in H68 of D. radiodurans LSU rRNA (PDB: 1NKW) (1).

\begin{tabular}{|c|c|c|c|c|}
\hline \multicolumn{5}{|c|}{$\begin{array}{l}\text { Hydrogen bond restraints } \\
1: \mathrm{G} 5{ }^{\prime} 1: \mathrm{H} 1 \\
1: \mathrm{C} 19: \mathrm{N} 3\end{array}$} \\
\hline $1: G 5^{\prime}-1: 06$ & $1: \mathrm{C}^{-} 19: \mathrm{H} 42$ & 1.800 & 2.500 & \\
\hline $1: \mathrm{G} 5^{\prime}{ }^{\prime} 1: \mathrm{H} 22$ & $1: \mathrm{C}^{-} 19: 02$ & 1.800 & 2.500 & \\
\hline $1: \mathrm{G} \_2: \mathrm{H} 1$ & $1: \mathrm{C}^{-} 18: \mathrm{N} 3$ & 1.800 & 2.500 & \\
\hline $1: \mathrm{G}^{-} 2: 06$ & $1: \mathrm{C}_{-} 18: \mathrm{H} 42$ & 1.800 & 2.500 & \\
\hline $1: \mathrm{G}^{-} 2: \mathrm{H} 22$ & $1: \mathrm{C}^{-} 18: 02$ & 1.800 & 2.500 & \\
\hline $1: \mathrm{G}^{-} 7: \mathrm{H1}$ & $1: \mathrm{C}^{-} 13: \mathrm{N} 3$ & 1.800 & 2.500 & \\
\hline $1: \mathrm{G}^{-} 7: 06$ & $1: \mathrm{C}^{-} 13: \mathrm{H} 42$ & 1.800 & 2.500 & \\
\hline $1: \mathrm{G}^{-} 7: \mathrm{H} 22$ & $1: \mathrm{C}^{-} 13: 02$ & 1.800 & 2.500 & \\
\hline $1: \mathrm{G}^{-} 8: \mathrm{H1}$ & $1: \mathrm{C}^{-} 12: \mathrm{N} 3$ & 1.800 & 2.500 & \\
\hline $1: G \_8: 06$ & $1: \mathrm{C}_{-} 12: \mathrm{H} 42$ & 1.800 & 2.500 & \\
\hline $1: \mathrm{G}^{-} 8: \mathrm{H} 22$ & $1: \mathrm{C}^{-} 12: \mathrm{O} 2$ & 1.800 & 2.500 & \\
\hline $1: \mathrm{C} \_9: \mathrm{N} 3$ & $1: \mathrm{G} 5{ }^{\prime} \_11: \mathrm{H} 1$ & 1.800 & 2.500 & \\
\hline $1: \mathrm{C}_{-} 9: \mathrm{H} 42$ & $1: G 5{ }^{\prime}-11: 06$ & 1.800 & 2.500 & \\
\hline $1: \mathrm{C}_{-} 9: \mathrm{O} 2$ & $1: \mathrm{G} 5 \mathrm{I}^{-} 11: \mathrm{H} 22$ & 1.800 & 2.500 & \\
\hline \multicolumn{5}{|c|}{ Distance restraints from SNOESY } \\
\hline $1: \mathrm{G} \_8: \mathrm{H} 1$ & $1: \mathrm{G} \_7: \mathrm{H} 1$ & 2.768 & 6.459 & \\
\hline $1: \mathrm{G}-4: \mathrm{H} 1$ & $1: \mathrm{U}_{-} 3: \mathrm{H} 3$ & 3.002 & 7.000 & 5.50 \\
\hline $1: \mathrm{G}-4: \mathrm{H} 1$ & $1: \mathrm{G} \_5: \mathrm{H} 1$ & 2.423 & 5.654 & 6.04 \\
\hline $1: \mathrm{G} \_5: \mathrm{H1}$ & $1: \mathrm{G}_{-}^{-} 14: \mathrm{H} 1$ & 2.500 & 7.000 & 7.82 \\
\hline
\end{tabular}




\begin{tabular}{|c|c|c|c|c|}
\hline $1: \mathrm{G} \_2: \mathrm{H} 1$ & $1: \mathrm{U} \_3: \mathrm{H} 3$ & 2.228 & 5.200 & \\
\hline $1: \mathrm{G}^{-} 2: \mathrm{H} 1$ & $1: \mathrm{G} \overline{5} ' \_1: \mathrm{H} 1$ & 3.079 & 7.000 & \\
\hline $1: \mathrm{U}_{-}^{-} 3: \mathrm{H} 3$ & $1: A \_1 \overline{7}: \mathrm{H} 2$ & 1.800 & 4.022 & 3.28 \\
\hline $1: \mathrm{U}^{-} 3: \mathrm{H} 3$ & $1: \mathrm{A}^{-} 17: \mathrm{H} 61$ & 1.964 & 4.582 & 3.84 \\
\hline $1: \mathrm{U}_{-} 3: \mathrm{H} 3$ & $1: A_{-} 17: \mathrm{H} 62$ & 1.800 & 4.102 & 2.46 \\
\hline $1: \mathrm{G}^{-} 4: \mathrm{H} 1$ & $1: \mathrm{A}^{-} 17: \mathrm{H} 61$ & 3.275 & 7.000 & 3.99 \\
\hline $1: \mathrm{G}^{-} 4: \mathrm{H} 1$ & $1: \mathrm{A}^{-} 17: \mathrm{H} 62$ & 2.467 & 5.757 & 3.46 \\
\hline $1: \mathrm{G} \overline{5}{ }^{\prime} \_1: \mathrm{H} 1$ & $1: \mathrm{P} \overline{3} ' \_20: \mathrm{H} 6$ & 3.102 & 7.000 & \\
\hline $1: \mathrm{C} \_1 \overline{9}: \mathrm{H} 41$ & $1:$ :3 ' $-20: \mathrm{H} 6$ & 2.081 & 4.857 & \\
\hline $1: \mathrm{C}^{-} 19: \mathrm{H} 42$ & $1: \mathrm{P} 3^{\prime}-20: \mathrm{H} 6$ & 1.846 & 4.308 & \\
\hline $1: \mathrm{G} \overline{5}{ }^{\prime} \_11: \mathrm{H} 1$ & $1: \mathrm{U}^{\prime}{ }^{\prime}-10: \mathrm{H} 1{ }^{\prime}$ & 2.428 & 5.664 & \\
\hline $1: 5^{\prime}{ }^{-} 1: \mathrm{H} 1$ & $1: \mathrm{P} 3^{\prime}-20: \mathrm{H} 1{ }^{\prime}$ & 2.062 & 4.811 & \\
\hline $1: \mathrm{G} \_2: \mathrm{H} 1$ & $1: \mathrm{U} 3 \overline{\mathrm{H}}: \mathrm{H}{ }^{\prime}$ & 2.204 & 5.144 & \\
\hline $1: \mathrm{U}_{-} 3: \mathrm{H} 3$ & $1: \mathrm{G}^{-} 4: \mathrm{H} 1^{\prime}$ & 2.369 & 5.528 & 3.57 \\
\hline $1: \mathrm{G}^{-} 2: \mathrm{H} 1$ & 1:C_19:H1' & 2.107 & 5.917 & \\
\hline $1: \mathrm{G} \overline{5}{ }^{\prime} \_1: \mathrm{H} 1$ & $1: \mathrm{G}^{-} 2: \mathrm{H} 1{ }^{\prime}$ & 2.368 & 5.526 & \\
\hline $1: \mathrm{G} \_7: \overline{\mathrm{H}} 1$ & $1: \mathrm{G}^{-} 14: \mathrm{H} 1^{\prime}$ & 2.040 & 4.761 & 3.58 \\
\hline $1: \mathrm{G}^{-} 5: \mathrm{H1}$ & $1: \mathrm{G}^{-} 14: \mathrm{H} 2^{\prime}$ & 2.413 & 5.631 & 7.07 \\
\hline $1: \mathrm{G}^{-} 14: \mathrm{H1}$ & $1: \mathrm{G} \_5: \mathrm{H} 1{ }^{\prime}$ & 1.800 & 7.000 & 10.76 \\
\hline \multicolumn{5}{|c|}{ Distance restraints fr̄om $\mathrm{D}_{2} \mathrm{O}$ NOESY } \\
\hline $1: G 5$ '_1:H8 & $1: \mathrm{G} 5$ ' $^{\prime} 1: \mathrm{H} 1{ }^{\prime}$ & 2.446 & 4.543 & \\
\hline $1: \mathrm{G}^{\prime}{ }^{-}-1: \mathrm{H} 8$ & $1: \mathrm{G} \_2: \mathrm{H} 8$ & 3.312 & 6.000 & \\
\hline $1: \mathrm{G}^{\prime}{ }^{\prime}-1: \mathrm{H} 1$ ' & $1: \mathrm{G} \overline{5} ' \_1: \mathrm{H} 3{ }^{\prime}$ & 2.528 & 4.696 & \\
\hline $1: \mathrm{G}^{\prime}{ }^{-}{ }^{1}$ : $\mathrm{H} 1$ ' & $1: \mathrm{G} 5$ ' $^{-} 1: \mathrm{H} 4{ }^{\prime}$ & 2.315 & 4.299 & \\
\hline 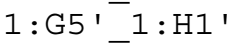 & $1: G \_2: H 8$ & 3.145 & 5.841 & \\
\hline $1: \mathrm{G}^{\prime}{ }^{\prime}-1: \mathrm{H} 2{ }^{\prime}$ & $1: \mathrm{G} \overline{5} '-1: \mathrm{H} 8$ & 3.123 & 5.800 & \\
\hline $1: \mathrm{G}^{\prime}{ }^{\prime}-1: \mathrm{H} 2{ }^{\prime}$ & $1: \mathrm{G}^{\prime}{ }^{\prime} \_1: \mathrm{H} 1{ }^{\prime}$ & 1.928 & 3.581 & \\
\hline $1: \mathrm{G}^{\prime}{ }^{\prime}-1: \mathrm{H} 2$ ' & $1: \mathrm{G} \_2: \mathrm{H} 8$ & 1.800 & 3.188 & \\
\hline $1: \mathrm{G}^{\prime}{ }^{\prime}-1: \mathrm{H} 2$ ' & $1: \mathrm{G}_{-} 2: \mathrm{H} 1{ }^{\prime}$ & 2.950 & 5.479 & \\
\hline $1: \mathrm{G}^{\prime}{ }^{\prime}{ }^{-} 1: \mathrm{H} 3^{\prime}$ & $1: \mathrm{G} \overline{5} '-1: \mathrm{H} 8$ & 2.256 & 4.190 & \\
\hline $1: \mathrm{G}^{\prime}{ }^{\prime}{ }^{-1: \mathrm{H}} 3^{\prime}$ & $1: \mathrm{G} \_2: \mathrm{H} 8$ & 2.078 & 3.860 & \\
\hline $1: G \_2: \mathrm{H} 8$ & $1: \mathrm{G}^{-} 2: \mathrm{H} \mathbf{C}^{\prime}$ & 2.689 & 4.994 & \\
\hline $1: \mathrm{G} \_2: \mathrm{H} 8$ & $1: \mathrm{G}^{-} 2: \mathrm{H} 3{ }^{\prime}$ & 2.238 & 4.157 & \\
\hline $1: \mathrm{G}^{-} 2: \mathrm{H}^{\prime}{ }^{\prime}$ & $1: \mathrm{G}^{-} 2: \mathrm{H}^{\prime}{ }^{\prime}$ & 2.530 & 4.699 & \\
\hline $1: \mathrm{G}^{-} 2: \mathrm{H} 1{ }^{\prime}$ & $1: \mathrm{G}^{-} 2: \mathrm{H} 4{ }^{\prime}$ & 2.567 & 4.768 & \\
\hline $1: \mathrm{G}^{-} 2: \mathrm{H} 2{ }^{\prime}$ & $1: \mathrm{G}^{-} 2: \mathrm{H} 8$ & 2.864 & 5.318 & \\
\hline $1: \mathrm{G}^{-} 2: \mathrm{H} 2{ }^{\prime}$ & $1: \mathrm{G}^{-} 2: \mathrm{H} 1^{\prime}$ & 2.128 & 3.952 & \\
\hline $1: \mathrm{G}^{-} 2: \mathrm{H} 3{ }^{\prime}$ & $1: \mathrm{U}_{-}^{-} 3: \mathrm{H} 6$ & 2.125 & 3.947 & \\
\hline $1: \mathrm{G}^{-} 2: \mathrm{H} 4{ }^{\prime}$ & $1: \mathrm{G} \_2: \mathrm{H} 8$ & 2.614 & 4.854 & \\
\hline $1: \mathrm{U}_{-} 3: \mathrm{H} 6$ & $1: \mathrm{G}^{-} 2: \mathrm{H} 2{ }^{\prime}$ & 1.806 & 3.354 & \\
\hline $1: \mathrm{U}_{-} 3: \mathrm{H} 6$ & $1: \mathrm{U}_{-} 3: \mathrm{H} 5$ & 1.800 & 3.162 & \\
\hline $1: \mathrm{U}^{-} 3: \mathrm{H} 6$ & $1: \mathrm{U}^{-} 3: \mathrm{H} 3{ }^{\prime}$ & 2.064 & 3.833 & 2.89 \\
\hline $1: \mathrm{U}_{-} 3: \mathrm{H} 1^{\prime}$ & $1: \mathrm{G}^{-} 2: \mathrm{H} 2{ }^{\prime}$ & 2.837 & 5.269 & \\
\hline $1: \mathrm{U}_{-} 3: \mathrm{H} 1^{\prime}$ & $1: \mathrm{U}_{-} 3: \mathrm{H} 6$ & 2.896 & 5.379 & 3.55 \\
\hline $1: \mathrm{U}_{-} 3: \mathrm{H1}{ }^{\prime}$ & $1: \mathrm{U}^{-} 3: \mathrm{H} 3{ }^{\prime}$ & 3.102 & 5.761 & 3.86 \\
\hline $1: \mathrm{U}_{-} 3: \mathrm{H} 2{ }^{\prime}$ & $1: \mathrm{U}^{-} 3: \mathrm{H1}{ }^{\prime}$ & 1.985 & 3.686 & 2.80 \\
\hline $1: \mathrm{U}_{-} 3: \mathrm{H} 3{ }^{\prime}$ & $1: \mathrm{G}_{-}^{-} 4: \mathrm{H} 8$ & 1.938 & 3.599 & 2.23 \\
\hline $1: \mathrm{G}^{-} 4: \mathrm{H} 8$ & $1: \mathrm{U}_{-} 3: \mathrm{H} 6$ & 3.334 & 6.000 & 4.44 \\
\hline $1: \mathrm{G} \_4: \mathrm{H} 8$ & $1: \mathrm{U}_{-} 3: \mathrm{H} 1^{\prime}$ & 3.432 & 6.000 & 4.76 \\
\hline $1: \mathrm{G}^{-} 4: \mathrm{H} 8$ & $1: \mathrm{U}^{-} 3: \mathrm{H} 2{ }^{\prime}$ & 1.800 & 3.342 & 2.15 \\
\hline $1: \mathrm{G} 4: \mathrm{H} 8$ & $1: \mathrm{G}^{\prime} 4: \mathrm{H} 1^{\prime}$ & 2.936 & 5.453 & 3.73 \\
\hline $1: \mathrm{G} 4: \mathrm{H} 8$ & $1: \mathrm{G} 44: \mathrm{H} 2{ }^{\prime}$ & 2.631 & 4.886 & 4.09 \\
\hline $1: \mathrm{G} 4: \mathrm{H} 8$ & $1: \mathrm{G}_{-} 4: \mathrm{H} 3{ }^{\prime}$ & 2.022 & 3.755 & 2.69 \\
\hline $1: \mathrm{G} 4: \mathrm{H} \mathbf{\prime}^{\prime}$ & $1: \mathrm{U}^{\prime} 3: \mathrm{H} 2{ }^{\prime}$ & 2.884 & 5.355 & 3.41 \\
\hline $1: \mathrm{G}^{-} 4: \mathrm{H} 1^{\prime}$ & $1: \mathrm{G}^{-} 4: \mathrm{H} 2{ }^{\prime}$ & 1.969 & 3.656 & 2.78 \\
\hline $1: \mathrm{G}_{-} 4: \mathrm{H} 1^{\prime}$ & $1: \mathrm{G}_{-} 4: \mathrm{H} 3{ }^{\prime}$ & 3.085 & 5.730 & 3.80 \\
\hline $1: \mathrm{G}_{-} 4: \mathrm{H} 1{ }^{\prime}$ & $1: \mathrm{G}_{-} 4: \mathrm{H} 4{ }^{\prime}$ & 2.741 & 5.091 & 3.53 \\
\hline
\end{tabular}




\begin{tabular}{|c|c|c|c|c|}
\hline $1: \mathrm{G} \_4: \mathrm{H} 1{ }^{\prime}$ & $1:$ A_1 $17: \mathrm{H} 2$ & 2.181 & 4.051 & 3.05 \\
\hline $1: \mathrm{G}^{-} 4: \mathrm{H} 2{ }^{\prime}$ & $1: \mathrm{G}^{-} 5: \mathrm{H} 8$ & 2.446 & 4.543 & 2.06 \\
\hline $1: \mathrm{G}^{-} 4: \mathrm{H}^{\prime}{ }^{\prime}$ & $1: \mathrm{G}^{-} 4: \mathrm{H} 2{ }^{\prime}$ & 1.927 & 3.578 & 2.44 \\
\hline $1: \mathrm{G}^{-} 4: \mathrm{H} 3{ }^{\prime}$ & $1: \mathrm{G}^{-} 5: \mathrm{H} 8$ & 2.170 & 4.029 & 3.6 \\
\hline $1: \mathrm{G}^{-} 4: \mathrm{H} 4{ }^{\prime}$ & $1: \mathrm{G}^{-} 4: \mathrm{H} 2{ }^{\prime}$ & 2.672 & 4.962 & 3.8 \\
\hline $1: \mathrm{G} \_5: \mathrm{H} 8$ & $1: \mathrm{G} \_5: \mathrm{H} 1^{\prime}$ & 3.002 & 5.574 & 3.7 \\
\hline $1: \mathrm{G} \_5: \mathrm{H} 8$ & $1: \mathrm{G} \_5: \mathrm{H} 2{ }^{\prime}$ & 1.800 & 2.778 & 4.0 \\
\hline $1: \mathrm{G} \_5: \mathrm{H} 1{ }^{\prime}$ & $1: \mathrm{G} \_5: \mathrm{H} 4{ }^{\prime}$ & 2.809 & 5.216 & 3.4 \\
\hline $1: \mathrm{G}^{-} 5: \mathrm{H} 2{ }^{\prime}$ & $1: \mathrm{G}^{-} 5: \mathrm{H} 1^{\prime}$ & 2.288 & 4.250 & 2.7 \\
\hline $1: \mathrm{G} \_5: \mathrm{H} 3{ }^{\prime}$ & $1: G \_5: H 8$ & 2.331 & 4.329 & 2.66 \\
\hline $1: \mathrm{A} \_6: \mathrm{H} 2$ & $1: \mathrm{G}_{-} 7: \mathrm{H} 1^{\prime}$ & 2.690 & 4.996 & 4.8 \\
\hline $1: \mathrm{A} \_6: \mathrm{H} 8$ & $1: \mathrm{G} \_5: \mathrm{H} 1^{\prime}$ & 2.329 & 4.325 & 5.3 \\
\hline $1:$ A_6 $6: \mathrm{H} 8$ & $1: \mathrm{A} \_6: \mathrm{H} 2^{\prime}$ & 2.934 & 5.449 & 3.8 \\
\hline $1: \mathrm{A} \_6: \mathrm{H} 8$ & $1: \mathrm{G} \_7: \mathrm{H} 8$ & 2.630 & 4.884 & 3.6 \\
\hline $1:$ A_6:H3' & $1: \mathrm{A} \_6: \mathrm{H8}$ & 2.116 & 3.929 & 2.6 \\
\hline $1: \mathrm{G} \_7: \mathrm{H} 8$ & $1: \mathrm{A} \_6: \mathrm{H} 2^{\prime}$ & 2.375 & 4.411 & 2.32 \\
\hline $1: \mathrm{G} \_7: \mathrm{H} 8$ & $1:$ A_6 :H3 ' & 1.800 & 5.000 & 2.59 \\
\hline $1: \mathrm{G} \_7: \mathrm{H} 8$ & $1: \mathrm{G}^{-} 7: \mathrm{H} 1^{\prime}$ & 1.954 & 5.500 & 3.4 \\
\hline $1: \mathrm{G}^{-} 7: \mathrm{H} 8$ & $1: \mathrm{G}^{-} 7: \mathrm{H} 3{ }^{\prime}$ & 1.800 & 3.310 & 3.7 \\
\hline $1: \mathrm{G} \_8: \mathrm{H} 8$ & $1: \mathrm{G}^{-} 7: \mathrm{H} 1^{\prime}$ & 3.095 & 5.748 & \\
\hline $1: \mathrm{G} \_8: \mathrm{H} 8$ & $1: \mathrm{G}^{-} 8: \mathrm{H} 1^{\prime}$ & 2.767 & 5.138 & \\
\hline $1: \mathrm{G} \_8: \mathrm{H} 8$ & $1: \mathrm{G}^{-} 8: \mathrm{H} 2{ }^{\prime}$ & 2.916 & 5.415 & \\
\hline $1: \mathrm{G}_{-} 8: \mathrm{H} 1{ }^{\prime}$ & $1: \mathrm{G}^{-} 8: \mathrm{H} 3^{\prime}$ & 2.291 & 4.255 & \\
\hline $1: \mathrm{G}_{-} 8: \mathrm{H} 1{ }^{\prime}$ & $1: \mathrm{G}^{-} 8: \mathrm{H} 4{ }^{\prime}$ & 2.367 & 4.395 & \\
\hline $1: \mathrm{G}_{-} 8: \mathrm{H} 2{ }^{\prime}$ & $1: \mathrm{G}^{-} 8: \mathrm{H} 1^{\prime}$ & 1.907 & 3.541 & \\
\hline $1: \mathrm{G}_{-} 8: \mathrm{H} 3{ }^{\prime}$ & $1: \mathrm{G} \_8: \mathrm{H} 8$ & 1.800 & 5.000 & \\
\hline $1:$ C_9 :H5 & $1: G_{-} 8: \mathrm{H} 2{ }^{\prime}$ & 2.761 & 5.127 & \\
\hline $1: \mathrm{C}_{-} 9: \mathrm{H} 6$ & $1: \mathrm{G}^{-} 8: \mathrm{H} 1^{\prime}$ & 3.251 & 6.000 & \\
\hline $1: \mathrm{C}_{-}^{-} 9: \mathrm{H} 6$ & $1: \mathrm{G}^{-} 8: \mathrm{H} 2{ }^{\prime}$ & 1.800 & 3.016 & \\
\hline $1: \mathrm{C}_{-} 9: \mathrm{H} 6$ & $1: \mathrm{G}^{-} 8: \mathrm{H} 3{ }^{\prime}$ & 1.811 & 5.000 & \\
\hline $1: \mathrm{C}_{2} 9: \mathrm{H} 6$ & $1: \mathrm{C}_{-} 9: \mathrm{H} 5$ & 1.800 & 3.111 & \\
\hline $1: \mathrm{C}^{-} 9: \mathrm{H} 6$ & $1: \mathrm{C}^{-} 9: \mathrm{H} 1{ }^{\prime}$ & 2.537 & 4.711 & \\
\hline $1: \mathrm{C}_{-} 9: \mathrm{H} 6$ & $1: \mathrm{C}_{-} 9: \mathrm{H} 3{ }^{\prime}$ & 1.800 & 3.312 & \\
\hline $1: \mathrm{C}^{-} 9: \mathrm{H} 6$ & $1: \mathrm{U} \overline{3} ' \_10: \mathrm{H} 6$ & 2.843 & 5.279 & \\
\hline $1: \mathrm{C} \_9: \mathrm{H} 1{ }^{\prime}$ & $1: G \_8: H 2{ }^{\prime}$ & 2.542 & 4.720 & \\
\hline $1: \mathrm{C}^{-} 9: \mathrm{H} 1{ }^{\prime}$ & $1: \mathrm{C}^{-} 9: \mathrm{H} 3^{\prime}$ & 2.678 & 4.973 & \\
\hline $1: \mathrm{C}_{-} 9: \mathrm{H} 1{ }^{\prime}$ & $1: \mathrm{U} 3{ }^{\prime} \_10: \mathrm{H} 6$ & 3.172 & 5.892 & \\
\hline 1:C_9 $9: \mathrm{H} 2{ }^{\prime}$ & $1: \mathrm{C} \_9: \mathrm{H} 1{ }^{\prime}$ & 1.935 & 3.594 & \\
\hline $1: \mathrm{C} \_9: \mathrm{H} 2{ }^{\prime}$ & $1: \mathrm{U3}{ }^{\prime} \_10: \mathrm{H} 5$ & 2.690 & 4.996 & \\
\hline $1: \mathrm{C}^{-} 9: \mathrm{H} 2{ }^{\prime}$ & $1: \mathrm{U} 3^{\prime}{ }^{\prime}-10: \mathrm{H} 6$ & 1.800 & 3.136 & \\
\hline $1: \mathrm{C}_{-} 9: \mathrm{H} 2{ }^{\prime}$ & $1: \mathrm{U} 3{ }^{\prime} \_10: \mathrm{H} 1{ }^{\prime}$ & 2.401 & 4.459 & \\
\hline $1: \mathrm{C}^{-} 9: \mathrm{H} 3{ }^{\prime}$ & $1: \mathrm{U} 3^{\prime}-10: \mathrm{H} 6$ & 2.372 & 4.405 & \\
\hline $1: \mathrm{C} \_9: \mathrm{H} 4{ }^{\prime}$ & $1: \mathrm{C}_{-} 9: \mathrm{H} 1{ }^{\prime}$ & 2.749 & 5.105 & \\
\hline $1: \mathrm{U} \overline{3}{ }^{\prime} \_10: \mathrm{H} 5$ & $1: \mathrm{U} \overline{3} ' \_10: \mathrm{H} 6$ & 1.800 & 3.194 & \\
\hline $1: \mathrm{U3}{ }^{\prime} \_10: \mathrm{H} 6$ & $1: \mathrm{U} 3{ }^{\prime} \_10: \mathrm{H} 1{ }^{\prime}$ & 2.792 & 5.185 & \\
\hline $1: \mathrm{U} 3{ }^{\prime} \_10: \mathrm{H} 2{ }^{\prime}$ & $1: \mathrm{U} 3{ }^{\prime} \_10: \mathrm{H} 6$ & 2.290 & 4.252 & \\
\hline $1: \mathrm{U} 3{ }^{\prime} \_10: \mathrm{H} 2{ }^{\prime}$ & $1: \mathrm{U} 3{ }^{\prime} \_10: \mathrm{H} 1{ }^{\prime}$ & 2.074 & 3.852 & \\
\hline $1: \mathrm{U} 3{ }^{\prime} \_10: \mathrm{H} 3{ }^{\prime}$ & $1: \mathrm{U} 3{ }^{\prime} \_10: \mathrm{H} 6$ & 1.873 & 3.478 & \\
\hline $1: \mathrm{U} 3{ }^{\prime} \_10: \mathrm{H} 3{ }^{\prime}$ & $1: \mathrm{U} 3{ }^{\prime} \_10: \mathrm{H} 1{ }^{\prime}$ & 2.560 & 4.754 & \\
\hline $1:$ G5 '_-11:H8 & $1: \mathrm{G} 5{ }^{\prime} \_11: \mathrm{H} 1{ }^{\prime}$ & 2.511 & 4.663 & \\
\hline $1: \mathrm{G} 5{ }^{\prime} \_11: \mathrm{H} 8$ & $1: \mathrm{G} 5{ }^{\prime} \_11: \mathrm{H} 4{ }^{\prime}$ & 2.890 & 5.367 & \\
\hline $1: \mathrm{G} 5 \mathrm{I}^{-}{ }^{-11: \mathrm{H} 1}$ ' & $1: \mathrm{G} 5{ }^{\prime} \_11: \mathrm{H} 3{ }^{\prime}$ & 2.480 & 4.605 & \\
\hline $1: \mathrm{G} 5$ '_11:H1' & $1: \mathrm{G} 5{ }^{\prime}{ }^{\prime} 11: \mathrm{H} 4{ }^{\prime}$ & 2.342 & 4.349 & \\
\hline $1: \mathrm{G} 5 \mathrm{C}^{-}-11: \mathrm{H1}$ ' & $1:$ C_1 $12: \mathrm{H} 6$ & 3.153 & 5.855 & \\
\hline $1: G 5{ }^{\prime} \_11: \mathrm{H} 2{ }^{\prime}$ & $1: \mathrm{G}^{\prime}{ }^{\prime} \_11: \mathrm{H} 8$ & 2.596 & 4.820 & \\
\hline $1:$ G5 '_11:H2 ' & $1: \mathrm{G} 5{ }^{\prime} \_11: \mathrm{H} 1{ }^{\prime}$ & 1.898 & 3.525 & \\
\hline $1:$ G5 '_11:H3' & $1: \mathrm{G} 5^{\prime}-11: \mathrm{H} 8$ & 2.404 & 4.465 & \\
\hline
\end{tabular}




\begin{tabular}{|c|c|c|c|c|}
\hline $1: \mathrm{G}^{\prime}{ }^{\prime} 11: \mathrm{H} 3^{\prime}$ & $1: \mathrm{C} \_12: \mathrm{H} 6$ & 1.860 & 3.454 & \\
\hline $1: C \_1 \overline{2}:$ H5 & $1: \bar{G}^{\prime}{ }^{\prime} 11: \mathrm{H} 2{ }^{\prime}$ & 2.729 & 5.068 & \\
\hline $1: \mathrm{C}^{-} 12: \mathrm{H} 5$ & $1: \mathrm{G}^{\prime}{ }^{-}-11: \mathrm{H} 3^{\prime}$ & 2.520 & 4.679 & \\
\hline $1: \mathrm{C}^{-} 12: \mathrm{H} 6$ & $1: \mathrm{G}^{\prime}{ }^{-}-11: \mathrm{H} 2{ }^{\prime}$ & 1.800 & 3.133 & \\
\hline $1: \mathrm{C}^{-} 12: \mathrm{H} 6$ & $1:$ C_1 $1 \overline{2}:$ H5 & 1.800 & 3.226 & \\
\hline $1: \mathrm{C}^{-} 12: \mathrm{H} 6$ & $1: \mathrm{C}_{-} 12: \mathrm{H} 1^{\prime}$ & 2.736 & 5.081 & \\
\hline $1: \mathrm{C}^{-} 12: \mathrm{H} 1^{\prime}$ & $1: \mathrm{G}^{\prime}{ }^{\prime} \quad 11: \mathrm{H} 2{ }^{\prime}$ & 3.024 & 5.616 & \\
\hline $1: \mathrm{C}^{-} 12: \mathrm{HI}^{\prime}$ & $1: \mathrm{C} \_1 \overline{3}: \mathrm{H} 6$ & 3.219 & 5.978 & \\
\hline $1: \mathrm{C}^{-} 12: \mathrm{H} 2{ }^{\prime}$ & 1: $\mathrm{C}^{-} 12: \mathrm{H}^{\prime}$ & 2.047 & 3.801 & \\
\hline $1: \mathrm{C}_{-}^{-} 12: \mathrm{H} 2{ }^{\prime}$ & $1: \mathrm{C}^{-} 13: \mathrm{H} 6$ & 1.800 & 3.052 & \\
\hline $1: \mathrm{C}^{-} 12: \mathrm{H}^{\prime}{ }^{\prime}$ & $1: \mathrm{C}^{-} 12: \mathrm{H} 6$ & 1.829 & 3.397 & \\
\hline $1: \mathrm{C}_{-}^{-} 12: \mathrm{H}^{\prime}{ }^{\prime}$ & $1: \mathrm{C}_{-}{ }^{-12}: \mathrm{H} 1^{\prime}$ & 2.409 & 4.473 & \\
\hline $1: \mathrm{C}^{-} 13: \mathrm{H} 6$ & $1: \mathrm{C}^{-} 12: \mathrm{H} 3^{\prime}$ & 2.448 & 4.545 & \\
\hline $1: \mathrm{C}^{-} 13: \mathrm{H} 6$ & $1: \mathrm{C}^{-} 13: \mathrm{H} 5$ & 1.822 & 3.383 & \\
\hline $1: \mathrm{C}^{-} 13: \mathrm{H} 6$ & $1: \mathrm{C}^{-} 13: \mathrm{H} 3^{\prime}$ & 2.279 & 4.233 & 3.07 \\
\hline $1: \mathrm{C}^{-} 13: \mathrm{H} 1^{\prime}$ & $1: \mathrm{C}^{-} 12: \mathrm{H} 2^{\prime}$ & 2.718 & 5.047 & \\
\hline $1: \mathrm{C}^{-} 13: \mathrm{H} 1^{\prime}$ & $1: \mathrm{C}^{-} 13: \mathrm{H} 6$ & 2.662 & 4.944 & 3.52 \\
\hline $1: \mathrm{C}^{-} 13: \mathrm{H} 1^{\prime}$ & $1: \mathrm{C}^{-} 13: \mathrm{H}^{\prime}{ }^{\prime}$ & 2.755 & 5.116 & 3.89 \\
\hline $1: \mathrm{C}^{-} 13: \mathrm{H}^{\prime}$ & $1: \mathrm{G}^{-} 14: \mathrm{H} 8$ & 3.394 & 6.000 & 5.24 \\
\hline $1: \mathrm{C}^{-} 13: \mathrm{H} 2{ }^{\prime}$ & $1: \mathrm{C}^{-} 13: \mathrm{H} 1^{\prime}$ & 1.986 & 3.689 & 2.80 \\
\hline $1: \mathrm{C}^{-} 13: \mathrm{H} 2{ }^{\prime}$ & $1: \mathrm{G}^{-} 14: \mathrm{H} 8$ & 2.130 & 3.956 & 2.87 \\
\hline $1: \mathrm{C}_{-} 13: \mathrm{H} 2{ }^{\prime}$ & $1: \mathrm{G} \_14: \mathrm{H}^{\prime}{ }^{\prime}$ & 2.577 & 4.787 & 3.27 \\
\hline $1: \mathrm{C}^{-} 13: \mathrm{H}^{\prime}{ }^{\prime}$ & $1: \mathrm{G}^{-} 14: \mathrm{H} 8$ & 2.121 & 3.938 & 2.21 \\
\hline $1: \mathrm{C}^{-} 13: \mathrm{H} 4{ }^{\prime}$ & $1: \mathrm{C}^{-} 13: \mathrm{H} 1^{\prime}$ & 2.741 & 5.091 & 3.29 \\
\hline $1: \mathrm{G}^{-} 14: \mathrm{H} 8$ & $1: \mathrm{G}^{-} 14: \mathrm{H} 3^{\prime}$ & 2.242 & 4.163 & 2.86 \\
\hline $1: \mathrm{G}^{-} 14: \mathrm{H} 1^{\prime}$ & $1: \mathrm{G}_{-1} 14: \mathrm{H} 3{ }^{\prime}$ & 3.099 & 5.755 & 3.87 \\
\hline $1: \mathrm{G}^{-} 14: \mathrm{H} 2{ }^{\prime}$ & $1: \mathrm{G}^{-} 14: \mathrm{H} 8$ & 2.060 & 3.826 & 3.99 \\
\hline $1: \mathrm{G}^{-} 14: \mathrm{H} 2{ }^{\prime}$ & $1: \mathrm{G} \_14: \mathrm{H} 1^{\prime}$ & 2.118 & 3.934 & 2.79 \\
\hline $1: \mathrm{G}^{-} 14: \mathrm{H} 2{ }^{\prime}$ & $1: \mathrm{A}^{-} 15: \mathrm{H} 8$ & 2.098 & 3.895 & 4.79 \\
\hline $1: \mathrm{A} \_15: \mathrm{H} 2$ & $1: A \_6: H 1 '$ & 2.496 & 4.636 & 10.23 \\
\hline $1: \mathrm{A}^{-} 15: \mathrm{H1}{ }^{\prime}$ & $1: \mathrm{A}^{-} 6: \mathrm{H} 2$ & 2.635 & 4.894 & 5.63 \\
\hline $1: \mathrm{A}^{-} 15: \mathrm{H} 1^{\prime}$ & 1:A_15:H2 ' & 2.357 & 4.377 & 2.79 \\
\hline $1: \mathrm{A}^{-} 15: \mathrm{H1}{ }^{\prime}$ & $1:$ A_1 $^{-} 15: \mathrm{H} 3^{\prime}$ & 2.666 & 4.951 & 3.81 \\
\hline $1: \mathrm{A}^{-} 15: \mathrm{H} 3^{\prime}$ & $1: \mathrm{A} \_15: \mathrm{H} 8$ & 2.505 & 4.652 & 1.91 \\
\hline $1: \mathrm{A}^{-} 16: \mathrm{H} 2$ & $1: \mathrm{A} \_17: \mathrm{H} 1^{\prime}$ & 2.350 & 4.364 & 4.37 \\
\hline $1: \mathrm{A}^{-} 16: \mathrm{H8}$ & $1:$ A_1 $^{-} 15: \mathrm{H} 2^{\prime}$ & 2.082 & 3.867 & 2.83 \\
\hline $1: \mathrm{A}^{-} 16: \mathrm{H} 8$ & $1: \mathrm{A}^{-} 15: \mathrm{H} 3^{\prime}$ & 2.534 & 4.706 & 3.86 \\
\hline $1: \mathrm{A}^{-} 16: \mathrm{H} 8$ & $1:$ A_1 $^{-16: \mathrm{H}^{\prime}}$ & 2.280 & 4.234 & 2.85 \\
\hline $1: \mathrm{A}^{-} 16: \mathrm{H} 8$ & $1: \mathrm{A} \_16: \mathrm{H} 4{ }^{\prime}$ & 3.047 & 5.660 & 4.39 \\
\hline $1: \mathrm{A}^{-} 16: \mathrm{H8}$ & $1: \mathrm{A} \_17: \mathrm{H} 8$ & 3.366 & 6.000 & 3.93 \\
\hline $1: \mathrm{A}^{-} 16: \mathrm{H} 1^{\prime}$ & $1: \mathrm{A}-15: \mathrm{H} 2$ & 2.172 & 4.035 & 10.78 \\
\hline $1: \mathrm{A}_{-} 16: \mathrm{H} 1^{\prime}$ & 1:A_15:H2 ' & 3.050 & 5.665 & 5.83 \\
\hline $1: \mathrm{A}^{-} 16: \mathrm{H} 1^{\prime}$ & $1: \mathrm{A}-16: \mathrm{H} 8$ & 2.868 & 5.326 & 3.81 \\
\hline $1: \mathrm{A}^{-} 16: \mathrm{H} 1^{\prime}$ & $1: \mathrm{A}^{-} 16: \mathrm{H} 2^{\prime}$ & 1.912 & 3.552 & 2.81 \\
\hline $1: \mathrm{A}^{-} 16: \mathrm{H} 1^{\prime}$ & $1:$ A_1 $^{-} 16: \mathrm{H} 3^{\prime}$ & 2.870 & 5.329 & 3.87 \\
\hline $1: \mathrm{A}^{-} 16: \mathrm{H} 1^{\prime}$ & $1: A_{-} 16: \mathrm{H}^{\prime}{ }^{\prime}$ & 2.475 & 4.596 & 3.25 \\
\hline $1: A \_16: \mathrm{H} 1^{\prime}$ & $1:$ A_1 $17: \mathrm{H} 8$ & 3.410 & 6.000 & 5.15 \\
\hline $1: \mathrm{A}^{-} 16: \mathrm{H} 2{ }^{\prime}$ & $1: \mathrm{A}^{-} 16: \mathrm{H} 8$ & 3.069 & 5.700 & 3.88 \\
\hline $1: \mathrm{A}_{-} 16: \mathrm{H} 2^{\prime}$ & $1: \mathrm{A} \_17: \mathrm{H} 8$ & 1.845 & 3.427 & 2.72 \\
\hline $1: \mathrm{A}^{-} 17: \mathrm{H} 2$ & 1: $\mathrm{C}_{-}^{-1} 18: \mathrm{H}^{\prime}$ & 2.440 & 4.532 & \\
\hline $1: \mathrm{A} \_17: \mathrm{H} 8$ & $1:$ A_17:H3 ' & 1.889 & 3.508 & 2.72 \\
\hline $1: \mathrm{A}^{-} 17: \mathrm{H} 8$ & $1: \mathrm{A}^{-} 16: \mathrm{H} 3^{\prime}$ & 1.800 & 5.000 & 2.62 \\
\hline $1: \mathrm{A} \_17: \mathrm{H} 8$ & $1:$ A_1 $17: \mathrm{H}^{\prime}$ & 2.886 & 5.359 & 3.80 \\
\hline $1: \mathrm{A}^{-} 17: \mathrm{H} 8$ & $1: \mathrm{C}^{-} 18: \mathrm{H} 5$ & 3.041 & 5.648 & \\
\hline $1: \mathrm{A}^{-} 17: \mathrm{H} 1^{\prime}$ & $1: \mathrm{A}^{-} 16: \mathrm{H} 2^{\prime}$ & 2.720 & 5.052 & 3.74 \\
\hline $1: \mathrm{A}^{-} 17: \mathrm{H} 1^{\prime}$ & 1:A_17:H3 ' & 2.776 & 5.156 & 3.85 \\
\hline $1: \mathrm{A}_{-} 17: \mathrm{H} 1^{\prime}$ & $1:$ A_17:H4 ' & 2.571 & 4.775 & 3.26 \\
\hline
\end{tabular}




\begin{tabular}{|c|c|c|c|c|}
\hline $1: \mathrm{A} \_17: \mathrm{H} 1^{\prime}$ & $1: \mathrm{C} \_18: \mathrm{H} 6$ & 2.816 & 5.229 & \\
\hline $1: A_{-} 17: \mathrm{H} 2{ }^{\prime}$ & $1: \mathrm{A} \_17: \mathrm{H} 1^{\prime}$ & 1.967 & 3.654 & 2.80 \\
\hline $1: \mathrm{A}^{-} 17: \mathrm{H} 3^{\prime}$ & $1: \mathrm{C}_{-} 18: \mathrm{H} 6$ & 2.353 & 4.371 & \\
\hline $1: \mathrm{C}^{-} 18: \mathrm{H} 6$ & $1: \mathrm{A} \_17: \mathrm{H} 2^{\prime}$ & 1.800 & 3.283 & \\
\hline $1: \mathrm{C}^{-} 18: \mathrm{H} 6$ & $1: \mathrm{C}_{-}^{-18} 18: \mathrm{H} 5$ & 1.800 & 3.188 & \\
\hline $1: \mathrm{C}^{-} 18: \mathrm{H} 6$ & $1: \mathrm{C}_{-} 18: \mathrm{H} 2^{\prime}$ & 2.932 & 5.444 & \\
\hline $1: C_{1} 18: \mathrm{H} 6$ & $1: \mathrm{C}_{2} 18: \mathrm{H} 3^{\prime}$ & 1.822 & 3.383 & \\
\hline $1: \mathrm{C}^{-} 18: \mathrm{H} 1^{\prime}$ & $1: \mathrm{A}_{-} 17: \mathrm{H} 2^{\prime}$ & 2.909 & 5.403 & \\
\hline $1: \mathrm{C}_{1} 18: \mathrm{H} 1^{\prime}$ & $1: \mathrm{C}_{-} 18: \mathrm{H} 3{ }^{\prime}$ & 2.102 & 3.904 & \\
\hline $1: \mathrm{C}^{-} 18: \mathrm{HI}^{\prime}$ & $1: \mathrm{C}_{-} 18: \mathrm{H} 4{ }^{\prime}$ & 2.537 & 4.711 & \\
\hline $1: \mathrm{C}_{1} 18: \mathrm{H} 2{ }^{\prime}$ & $1: C_{-} 18: \mathrm{H}^{\prime}$ & 1.921 & 3.567 & \\
\hline $1: \mathrm{C}^{-} 18: \mathrm{H}^{\prime}{ }^{\prime}$ & $1: \mathrm{C}_{-}^{-19} 19: \mathrm{H} 6$ & 2.100 & 3.900 & \\
\hline $1:$ C_19:H5 & $1: C_{-} 18: \mathrm{H} 2^{\prime}$ & 2.716 & 5.045 & \\
\hline $1: \mathrm{C}^{-} 19: \mathrm{H} 5$ & $1: \mathrm{C}_{-} 18: \mathrm{H}^{\prime}{ }^{\prime}$ & 2.566 & 4.766 & \\
\hline $1:$ C_19:H6 & $1: C_{-} 18: \mathrm{H}^{\prime}{ }^{\prime}$ & 1.800 & 3.117 & \\
\hline $1: \mathrm{C}^{-} 19: \mathrm{H} 6$ & $1: \mathrm{C}_{-1} 19: \mathrm{H} 5$ & 1.800 & 3.252 & \\
\hline $1: \mathrm{C}_{-} 19: \mathrm{H} 6$ & $1:$ C_1 $^{19: \mathrm{H}^{\prime}}$ & 2.675 & 4.968 & \\
\hline $1: \mathrm{C}^{-} 19: \mathrm{H} 1^{\prime}$ & 1: $\mathrm{C}_{-}{ }^{-18}: \mathrm{H} 2^{\prime}$ & 3.024 & 5.616 & \\
\hline 1:C_19:H1' & $1: \mathrm{C}_{-} 19: \mathrm{H}^{\prime}$ & 2.762 & 5.130 & \\
\hline $1: \mathrm{C}^{-} 19: \mathrm{H} 1^{\prime}$ & $1: \mathrm{P} \overline{3} ' \_20: \mathrm{H} 8$ & 3.380 & 6.000 & \\
\hline 1:C_19:H2 ' & $1:$ C_1 $19: \mathrm{H} 6$ & 2.436 & 5.500 & \\
\hline $1: \mathrm{C}^{-} 19: \mathrm{H} 2{ }^{\prime}$ & 1: C_19:H1' & 1.841 & 3.418 & \\
\hline 1:C_19:H2 ' & $1: \mathrm{P} 3{ }^{\prime} \_20: \mathrm{H} 8$ & 1.804 & 3.351 & \\
\hline $1: \mathrm{C}^{-} 19: \mathrm{H}^{\prime}{ }^{\prime}$ & $1:$ C_1 $19: \mathrm{H} 6$ & 1.800 & 3.328 & \\
\hline 1:C_19:H3 ' & $1: \mathrm{P} 3{ }^{\prime} \_20: \mathrm{H} 8$ & 2.190 & 4.067 & \\
\hline $1:$ C_19:H4 ' & $1: \mathrm{C} \_1 \overline{9}: \mathrm{H} 1^{\prime}$ & 2.731 & 5.073 & \\
\hline $1: \mathrm{P} \bar{\prime}{ }^{\prime} \_20: \mathrm{H} 8$ & $1: \mathrm{P} \overline{3}{ }^{\prime} \_20: \mathrm{H} 1^{\prime}$ & 2.699 & 5.013 & \\
\hline $1: \mathrm{P} 3^{\prime} \_20: \mathrm{H} 1^{\prime}$ & $1:$ P3 '_20:H3 ' & 2.703 & 5.020 & \\
\hline $1:$ P3 '_20:H1' & $1:$ P3 '_2 $0: \mathrm{H} 4{ }^{\prime}$ & 2.633 & 4.890 & \\
\hline $1:$ P3 '_20:H2 ' & $1:$ :3 ' $20: \mathrm{H} 8$ & 2.770 & 5.144 & \\
\hline $1:$ : $3{ }^{\prime} \_20: H 2{ }^{\prime}$ & $1: \mathrm{P} 3{ }^{\prime}{ }^{\prime} 20: \mathrm{H} 1^{\prime}$ & 2.109 & 3.916 & \\
\hline $1:$ :3' ${ }^{\prime} 20: \mathrm{H}^{\prime}$ & $1: \mathrm{P} 3{ }^{\prime}-20: \mathrm{H} 8$ & 1.929 & 3.583 & \\
\hline $1:$ : 3 '_20:H4 ' & $1:$ :3 '_20:H8 & 2.963 & 5.502 & \\
\hline
\end{tabular}


Table S2. List of Distance Restraints (in $\AA$ ) Used for the Structural Modeling of $\underset{\text { PCCG }}{\text { GAG } C C G}$. See text for Dihedral Angle Restraints.

\begin{tabular}{|c|c|c|c|}
\hline \multicolumn{4}{|c|}{ Hydrogen bond restraints } \\
\hline $1:$ G5 ' $1: \mathrm{H} 1$ & $1: \mathrm{C} \quad 19: \mathrm{N} 3$ & 1.800 & 2.500 \\
\hline $1:$ G5'_1:06 & $1:$ C_19:H42 & 1.800 & 2.500 \\
\hline $1: \mathrm{G}^{\prime} \mathrm{I}^{-1} 1: \mathrm{H} 22$ & $1: \mathrm{C}^{-} 19: 02$ & 1.800 & 2.500 \\
\hline $1: \mathrm{G} \quad \overline{\mathrm{H}} \mathrm{H} 1$ & $1: \mathrm{C}^{-} 18: \mathrm{N} 3$ & 1.800 & 2.500 \\
\hline $1: G \_2: 06$ & $1: \mathrm{C}-18: \mathrm{H} 42$ & 1.800 & 2.500 \\
\hline $1: G \_2: H 22$ & $1: C \_18: 02$ & 1.800 & 2.500 \\
\hline $1: \mathrm{G}^{-} 7: \mathrm{H} 1$ & $1: \mathrm{C}^{-} 13: \mathrm{N} 3$ & 1.800 & 2.500 \\
\hline $1: G^{-7} 7: 06$ & $1: \mathrm{C} \_13: \mathrm{H} 42$ & 1.800 & 2.500 \\
\hline $1: \mathrm{G}_{-}^{-} 7: \mathrm{H} 22$ & $1: \mathrm{C}^{-} 13: 02$ & 1.800 & 2.500 \\
\hline $1: \mathrm{G}^{-} 8: \mathrm{H} 1$ & $1: \mathrm{C}^{-} 12: \mathrm{N} 3$ & 1.800 & 2.500 \\
\hline $1: G \_8: 06$ & $1:$ C_12:H42 & 1.800 & 2.500 \\
\hline $1: G \_8: H 22$ & $1:$ C_12:O2 & 1.800 & 2.500 \\
\hline $1: \mathrm{C}^{-} 9: \mathrm{N} 3$ & $1: \mathrm{G}^{\prime} \mathrm{\prime} 11: \mathrm{H} 1$ & 1.800 & 2.500 \\
\hline $1:$ C_9:H4 2 & $1: G 51-11: 06$ & 1.800 & 2.500 \\
\hline $1:$ C_9:02 & $1:$ G5 ' $11: \mathrm{H} 22$ & 1.800 & 2.500 \\
\hline Dist̄ance re & Es from SNOESY & & \\
\hline $1:$ G_7 $:$ H1 & $1: G \_14: \mathrm{H} 1{ }^{\prime}$ & 2.153 & 4.000 \\
\hline $1: \mathrm{G}^{-1} 17: \mathrm{HI}$ & $1: \mathrm{G}^{-} 4: \mathrm{HI} \mathrm{I}^{\prime}$ & 1.800 & 5.000 \\
\hline $1: \mathrm{G}^{-} 2: \mathrm{H} 1$ & $1: \mathrm{U}^{-} 3: \mathrm{H} 3$ & 1.888 & 4.406 \\
\hline $1: \mathrm{G} \_2: \mathrm{H} 1$ & $1: \mathrm{G} \_17: \mathrm{H} 1$ & 2.081 & 4.855 \\
\hline $1: \mathrm{P} \overline{3}, \quad 20: \mathrm{H} 6$ & $1: G \overline{5}, \quad 1: \mathrm{H} 1$ & 2.184 & 5.097 \\
\hline $1:$ U_3: H3 & $1:$ C_1 $1 \overline{8}: \mathrm{H} 42$ & 2.291 & 5.347 \\
\hline $1: \mathrm{U} \_3: \mathrm{H} 3$ & $1:$ C_18:H41 & 2.638 & 6.156 \\
\hline $1:$ C_19:H42 & $1: \mathrm{P} \overline{3} ' \_20: \mathrm{H} 6$ & 2.363 & 5.514 \\
\hline $1: \mathrm{P} \overline{3} ' \_20: \mathrm{H} 6$ & $1: C \_1 \overline{9}:$ H4 1 & 1.900 & 4.433 \\
\hline $1: \mathrm{G}^{\prime}{ }^{-} 1: \mathrm{H1}$ & $1: \mathrm{P} \overline{3} ' 20: \mathrm{H} 1^{\prime}$ & 2.120 & 4.946 \\
\hline Distañce re & is from $\mathrm{D}_{2} \mathrm{O} \mathrm{NO}$ & & \\
\hline $1:$ A_6:H1' & $1:$ A_15:H2 & 1.800 & $4.857^{\circ}$ \\
\hline $1:$ A_16:H1' & $1:$ A_15:H2 & 1.800 & $4.299^{\circ}$ \\
\hline $1: \mathrm{A}^{-} 15: \mathrm{H} 1^{\prime}$ & $1: \mathrm{A}^{-} 6: \mathrm{H} 2$ & 1.800 & $4.140^{\circ}$ \\
\hline $1: \mathrm{A}_{-15} 15: \mathrm{H} 8$ & $1: \mathrm{A} \_5: \mathrm{H} 2$ & 2.000 & $5.000^{2}$ \\
\hline $1: \mathrm{G}^{-} 14: \mathrm{H} 1^{\prime}$ & $1: \mathrm{A}^{-} 5: \mathrm{H} 2$ & 1.800 & $5.000^{\circ}$ \\
\hline $1: \mathrm{G}^{-} 14: \mathrm{H} 2{ }^{\prime}$ & $1: \mathrm{A}^{-} 5: \mathrm{H} 2$ & 2.000 & $5.000^{\circ}$ \\
\hline $1: \mathrm{G}^{-} 14: \mathrm{H} 1^{\prime}$ & $1: \mathrm{A}-15: \mathrm{H} 8$ & 2.000 & $5.495^{\circ}$ \\
\hline $1:$ A_16:H8 & $1:$ A_15:H2' & 1.800 & $3.990^{\circ}$ \\
\hline $1: \mathrm{A}^{-} 6: \mathrm{H} 8$ & $1: \mathrm{A}^{-} 5: \mathrm{H} 1^{\prime}$ & 1.800 & $4.119^{a}$ \\
\hline $1: G_{-}^{\prime} 4:$ H3 ' & $1: \mathrm{A}_{-}^{-} 5: \mathrm{H} 8$ & 1.800 & $5.000^{2}$ \\
\hline $1: \mathrm{A}^{-} \quad 16: \mathrm{H} 8$ & $1: \mathrm{A}^{-} 15: \mathrm{H}^{\prime}$ & 1.800 & $5.000^{\circ}$ \\
\hline $1: G_{-}^{-} 4: \mathrm{H} 8$ & $1: A_{-}^{-} 5: \mathrm{H} 8$ & 3.401 & $6.000^{\circ}$ \\
\hline $1: \mathrm{A} 15: \mathrm{H} 8$ & $1: \mathrm{A}-16: \mathrm{H} 8$ & 3.084 & $5.727^{\circ}$ \\
\hline $1: \mathrm{A}^{-} 16: \mathrm{H} 2$ & $1: \mathrm{A}^{-} 5: \mathrm{H}^{\prime} \mathrm{\prime}$ & 2.834 & $5.262^{2}$ \\
\hline $1: \mathrm{A} 6: \mathrm{HI}$ & $1: \mathrm{A}^{-} 5: \mathrm{H} 2$ & 1.800 & $5.000^{\mathrm{b}}$ \\
\hline $1: \mathrm{A} \_16: \mathrm{H1}$ & $1: \mathrm{A} 5: \mathrm{H} 2$ & 1.800 & $5.000^{\mathrm{b}}$ \\
\hline $1: \mathrm{A}^{-} 5: \mathrm{H1}$ & $1: \mathrm{A}^{-} 16: \mathrm{H} 2$ & 1.800 & $5.000^{\mathrm{b}}$ \\
\hline $1: \mathrm{A} \_5: \mathrm{H} 8$ & $1: \mathrm{A}_{-}^{-} 15: \mathrm{H} 2$ & 1.800 & $5.000^{\mathrm{b}}$ \\
\hline $1: G \_4: H 1{ }^{\prime}$ & $1: \mathrm{A} \_15: \mathrm{H} 2$ & 1.800 & $5.000^{b}$ \\
\hline $1: \mathrm{G}^{-} 4: \mathrm{H} 2 \mathrm{I}^{\prime}$ & $1: \mathrm{A}^{-} 15: \mathrm{H} 2$ & 1.800 & $5.000^{\mathrm{b}}$ \\
\hline $1: A \_5: H 8$ & $1: \mathrm{G}^{-} 4: \mathrm{H1} \mathrm{I}^{\prime}$ & 1.800 & $5.516^{\mathrm{b}}$ \\
\hline $1: \mathrm{A}^{-} 6: \mathrm{H} 8$ & $1: \mathrm{A}^{-} 5: \mathrm{H} 2{ }^{\prime}$ & 1.800 & $4.658^{\mathrm{b}}$ \\
\hline $1: \mathrm{A}^{-} 15: \mathrm{H} 1{ }^{\prime}$ & $1: A \_16: \mathrm{H} 8$ & 1.800 & $4.340^{\mathrm{b}}$ \\
\hline
\end{tabular}




\begin{tabular}{|c|c|c|c|}
\hline $1:$ A_15:H8 & $1: \mathrm{G} \_14: \mathrm{H} 3{ }^{\prime}$ & 1.800 & $5.000^{\mathrm{b}}$ \\
\hline $1: \mathrm{A}^{-} 5: \mathrm{H} 3{ }^{\prime}$ & $1: \mathrm{A}^{-} 6: \mathrm{H} 8$ & 1.800 & $4.907^{b}$ \\
\hline $1: \mathrm{A}^{-} 6: \mathrm{H} 8$ & $1: \mathrm{A}^{-} 5: \mathrm{H} 8$ & 3.270 & $6.000^{\mathrm{b}}$ \\
\hline $1: \mathrm{A}^{-} 6: \mathrm{H} 2$ & $1: A_{-15} 154^{\prime}$ & 3.000 & $6.000^{\mathrm{b}}$ \\
\hline $1: \mathrm{A} \_5: \mathrm{H} 8$ & $1: \mathrm{G}^{-} 4: \mathrm{H} 2{ }^{\prime}$ & 1.800 & 5.000 \\
\hline $1: \mathrm{A} \_5: \mathrm{H} 8$ & $1: \mathrm{A} \_5: \mathrm{H} 1{ }^{\prime}$ & 1.800 & 4.965 \\
\hline $1: \mathrm{A} \_5: \mathrm{H} 8$ & $1: \mathrm{A} \_5: \mathrm{H} 2^{\prime}$ & 1.800 & $3.191^{\mathrm{C}}$ \\
\hline $1: A^{-} 5: \mathrm{H} 2^{\prime}$ & $1: \mathrm{A} \_5: \mathrm{H} 1{ }^{\prime}$ & 1.800 & 3.938 \\
\hline $1: \mathrm{A}^{-} 5: \mathrm{H} 3^{\prime}$ & $1: \mathrm{A}^{-} 5: \mathrm{H} 1^{\prime}$ & 1.800 & 4.810 \\
\hline $1: \mathrm{A}^{-} 5: \mathrm{H} 3^{\prime}$ & $1: \mathrm{A}^{-} 5: \mathrm{H} 8$ & 1.800 & 5.000 \\
\hline $1: A^{-} 5: \mathrm{H} 4{ }^{\prime}$ & $1: \mathrm{A} \_5: \mathrm{H} 1^{\prime}$ & 1.800 & 4.411 \\
\hline $1: \mathrm{A}^{-} 15: \mathrm{H} 8$ & $1: \mathrm{A} \_15: \mathrm{H} 1^{\prime}$ & 1.800 & 5.043 \\
\hline $1: \mathrm{A}^{-} 15: \mathrm{H8}$ & $1:$ A_1 $^{-} 15: \mathrm{H} 2^{\prime}$ & 1.800 & 5.000 \\
\hline $1: \mathrm{A} \_15: \mathrm{H} 8$ & 1:A_15:H3 ' & 1.800 & 4.265 \\
\hline $1: \mathrm{A}^{-} 15: \mathrm{H} \mathbf{\prime}^{\prime}$ & 1:A_15:H3 ' & 1.800 & 5.000 \\
\hline $1: \mathrm{A}^{-} 15: \mathrm{H} \mathbf{1}^{\prime}$ & $1: A_{-} 15: \mathrm{H} 4{ }^{\prime}$ & 1.800 & 4.420 \\
\hline $1: \mathrm{A}^{-} 15: \mathrm{H} 2{ }^{\prime}$ & $1:$ A_1 $^{-15: \mathrm{H} 1^{\prime}}$ & 1.800 & 3.646 \\
\hline $1: \mathrm{G}^{-} 14: \mathrm{H} 2{ }^{\prime}$ & $1: \mathrm{A}_{-} 15: \mathrm{H} 8$ & 1.800 & 4.762 \\
\hline $1: G \overline{5}{ }^{\prime} \_1: \mathrm{H} 8$ & $1: \mathrm{G} \overline{5}{ }^{\prime}{ }^{\prime} 1: \mathrm{H} 1^{\prime}$ & 2.637 & 4.897 \\
\hline $1: \mathrm{G} 5{ }^{\prime}-1: \mathrm{H} 8$ & $1: \mathrm{G}^{\prime}{ }^{\prime} \_1: \mathrm{H} 2^{\prime}$ & 2.645 & 4.911 \\
\hline $1: \mathrm{G} 5{ }^{\prime} \_1: \mathrm{H} 8$ & $1: \mathrm{G} 5{ }^{\prime} \_1: \mathrm{H} 4{ }^{\prime}$ & 2.863 & 5.318 \\
\hline $1: \mathrm{G}^{\prime}{ }^{\prime}-1: \mathrm{H} 1^{\prime}$ & $1: \mathrm{G}^{\prime}{ }^{\prime}-1: \mathrm{H} 3^{\prime}$ & 2.531 & 4.700 \\
\hline $1: \mathrm{G}^{\prime}{ }^{\prime}{ }^{-1}$ : $\mathrm{H} 1{ }^{\prime}$ & $1: \mathrm{G}^{\prime}{ }^{\prime}{ }^{-} 1: \mathrm{H} 4{ }^{\prime}$ & 2.506 & 4.654 \\
\hline $1: \mathrm{G}^{\prime}{ }^{\prime}{ }^{-1}$ : $\mathrm{H1}{ }^{\prime}$ & $1: \mathrm{G} \_2: \mathrm{H} 8$ & 2.872 & 5.334 \\
\hline $1: \mathrm{G} 5{ }^{\prime} \_1: \mathrm{H} 2^{\prime}$ & $1: \mathrm{G} 5{ }^{\prime} \_1: \mathrm{H} 1{ }^{\prime}$ & 1.936 & 3.596 \\
\hline $1: \mathrm{G}^{\prime}{ }^{\prime}-1: \mathrm{H} 2{ }^{\prime}$ & $1: \mathrm{G} \_2: \mathrm{H} 8$ & 1.800 & 3.191 \\
\hline $1: \mathrm{G}^{\prime}{ }^{\prime} 1: \mathrm{H} 3^{\prime}$ & $1: \mathrm{G} 5{ }^{\prime} \_1: \mathrm{H} 8$ & 2.352 & 4.367 \\
\hline $1: G \_2: H 8$ & $1: \mathrm{G}^{\prime}{ }^{\prime}{ }^{-} 1: \mathrm{H} 3{ }^{\prime}$ & 1.988 & 3.692 \\
\hline $1: \mathrm{G} \_2: \mathrm{H} 8$ & $1: \mathrm{G} 5{ }^{\prime}-1: \mathrm{H} 8$ & 3.259 & 6.000 \\
\hline $1: \mathrm{G}^{-} 2: \mathrm{H} 8$ & $1: \mathrm{G} \_2: \mathrm{H} 1{ }^{\prime}$ & 2.625 & 4.875 \\
\hline $1: \mathrm{G} \_2: \mathrm{H} 8$ & $1: \mathrm{G} \_2: \mathrm{H} 2{ }^{\prime}$ & 2.983 & 5.540 \\
\hline $1: \mathrm{G}^{-} 2: \mathrm{H} 8$ & $1: \mathrm{G}^{-} 2: \mathrm{H} 3{ }^{\prime}$ & 2.303 & 4.278 \\
\hline $1: \mathrm{G} \_2: \mathrm{H} 8$ & $1: \mathrm{U}_{-} 3: \mathrm{H} 5$ & 2.759 & 5.124 \\
\hline $1: \mathrm{G} \_2: \mathrm{H} 1{ }^{\prime}$ & $1: \mathrm{G} \overline{5}{ }^{\prime}{ }^{\prime} 1: \mathrm{H} 2{ }^{\prime}$ & 3.001 & 5.573 \\
\hline $1: \mathrm{G}^{-} 2: \mathrm{H} 1^{\prime}$ & $1: \mathrm{G} \_2: \mathrm{H} 2{ }^{\prime}$ & 1.889 & 3.508 \\
\hline $1: \mathrm{G}^{-} 2: \mathrm{H} 1^{\prime}$ & $1: \mathrm{G}^{-} 2: \mathrm{H} 3{ }^{\prime}$ & 2.891 & 5.369 \\
\hline $1: \mathrm{G} \_2: \mathrm{H} 2^{\prime}$ & $1: \mathrm{U}_{-} 3: \mathrm{H} \mathbf{1}^{\prime}$ & 2.486 & 4.617 \\
\hline $1: \mathrm{G}^{-} 2: \mathrm{H} 3^{\prime}$ & $1: \mathrm{U}_{-} 3: \mathrm{H} 6$ & 2.140 & 3.974 \\
\hline $1: G \_2: H 4{ }^{\prime}$ & $1: G \_2: H 8$ & 2.594 & 4.817 \\
\hline $1: \mathrm{G}^{-} 2: \mathrm{H} 4{ }^{\prime}$ & $1: \mathrm{G}^{-} 2: \mathrm{H} 1^{\prime}$ & 2.769 & 5.143 \\
\hline $1: \mathrm{U}_{-} 3: \mathrm{H} 5$ & $1: G \_2: H 2{ }^{\prime}$ & 2.574 & 4.780 \\
\hline $1: \mathrm{U}_{-} 3: \mathrm{H} 6$ & $1: \mathrm{G}^{-} 2: \mathrm{H} \mathbf{1}^{\prime}$ & 2.962 & 5.501 \\
\hline $1: \mathrm{U}_{-} 3: \mathrm{H} 6$ & $1: G \_2: H 2{ }^{\prime}$ & 1.847 & 3.430 \\
\hline $1: \mathrm{U}_{-} 3: \mathrm{H} 6$ & $1: U_{-} 3: \mathrm{H} 5$ & 1.800 & 3.329 \\
\hline $1: \mathrm{U}_{-} 3: \mathrm{H} 6$ & $1: \mathrm{U}_{-} 3: \mathrm{H} 1^{\prime}$ & 2.401 & 4.458 \\
\hline $1: \mathrm{U}_{-} 3: \mathrm{H} 6$ & $1: \mathrm{U}_{-} 3: \mathrm{H} 3{ }^{\prime}$ & 2.161 & 4.013 \\
\hline $1: \mathrm{U} \_3: \mathrm{H} 1{ }^{\prime}$ & $1: \mathrm{U}_{-} 3: \mathrm{H} 3{ }^{\prime}$ & 2.903 & 5.391 \\
\hline $1: \mathrm{U}_{-} 3: \mathrm{H} 1^{\prime}$ & $1: \mathrm{U}_{-} 3: \mathrm{H} 4{ }^{\prime}$ & 2.604 & 4.836 \\
\hline $1: \mathrm{U}_{-} 3: \mathrm{H} 2{ }^{\prime}$ & $1: \mathrm{U}_{-} 3: \mathrm{H} 6$ & 2.325 & 5.000 \\
\hline $1: \mathrm{U}_{-} 3: \mathrm{H} 2{ }^{\prime}$ & $1: \mathrm{U}_{-} 3: \mathrm{H} 1^{\prime}$ & 1.954 & 3.629 \\
\hline $1: \mathrm{U}_{-} 3: \mathrm{H} 2{ }^{\prime}$ & $1: \mathrm{G} \_4: \mathrm{H} 1^{\prime}$ & 2.373 & 4.408 \\
\hline $1: \mathrm{U} \_3: \mathrm{H} 4{ }^{\prime}$ & $1: \mathrm{U}_{-} 3: \mathrm{H} 6$ & 2.590 & 4.811 \\
\hline $1: \mathrm{G} \_4: \mathrm{H} 8$ & $1: \mathrm{U}_{-} 3: \mathrm{H} 6$ & 3.032 & 5.632 \\
\hline $1: \mathrm{G} \_4: \mathrm{H} 8$ & $1: \mathrm{U}_{-} 3: \mathrm{H} 1^{\prime}$ & 3.252 & 6.000 \\
\hline $1: G \_4: H 8$ & $1: \mathrm{U}_{-} 3: \mathrm{H} 2^{\prime}$ & 1.883 & 3.497 \\
\hline $1: \mathrm{G} \_4: \mathrm{H} 8$ & $1: \mathrm{U}_{-} 3: \mathrm{H} 3{ }^{\prime}$ & 1.942 & 3.606 \\
\hline $1: \mathrm{G} \_4: \mathrm{H} 1^{\prime}$ & $1: \mathrm{G} \_4: \mathrm{H} 8$ & 2.747 & 5.102 \\
\hline
\end{tabular}




\begin{tabular}{|c|c|c|c|}
\hline $1: \mathrm{G} \_4: \mathrm{H} 1^{\prime}$ & $1: \mathrm{G} \_4: \mathrm{H} 2{ }^{\prime}$ & 2.149 & 3.990 \\
\hline $1: \mathrm{G}^{-} 4: \mathrm{H} 1^{\prime}$ & $1: \mathrm{G}^{-} 4: \mathrm{H} 4{ }^{\prime}$ & 2.626 & 4.877 \\
\hline $1: \mathrm{G}^{-} 4: \mathrm{H} 2{ }^{\prime}$ & $1: \mathrm{G}_{-}^{-} 4: \mathrm{H} 8$ & 2.482 & 4.610 \\
\hline $1: \mathrm{G}^{-} 4: \mathrm{H} 3{ }^{\prime}$ & $1: \mathrm{G}^{-} 4: \mathrm{H} 8$ & 1.988 & 3.692 \\
\hline $1: \mathrm{G}^{-} 4: \mathrm{H} 3{ }^{\prime}$ & $1: \mathrm{G}^{-} 4: \mathrm{HI}$ & 2.586 & 4.803 \\
\hline $1: \mathrm{A} \_6: \mathrm{H} 8$ & $1: \mathrm{A} \_6: \mathrm{H} 1^{\prime}$ & 2.569 & 5.500 \\
\hline $1: A^{-} 6: \mathrm{H} 8$ & $1:$ A_ $^{-} 6: \mathrm{H}^{\prime}$ & 2.146 & 3.985 \\
\hline $1: A_{-} 6: \mathrm{H} 1^{\prime}$ & $1: \mathrm{A} \_6: \mathrm{H} 2^{\prime}$ & 2.665 & 4.950 \\
\hline $1: A_{-} 6: \mathrm{H} 1^{\prime}$ & $1: \mathrm{A}^{-} 6:$ : $3^{\prime}$ & 3.017 & 5.602 \\
\hline $1: A_{-} 6: \mathrm{H} 2^{\prime}$ & $1:$ A_6:H8 & 2.691 & 4.998 \\
\hline $1: A_{-} 6:$ H3 ' & $1: \mathrm{G}^{-} 7: \mathrm{H} 8$ & 1.800 & 3.321 \\
\hline $1: G_{-} 7: \mathrm{H} 8$ & $1:$ A_6:H8 & 2.477 & 4.600 \\
\hline $1: \mathrm{G} \_7: \mathrm{H} 8$ & $1: A \_6: \mathrm{H} 1^{\prime}$ & 3.796 & 6.000 \\
\hline $1: \mathrm{G} \_7: \mathrm{H} 8$ & $1: \mathrm{A} \_6: \mathrm{H} 2^{\prime}$ & 2.983 & 5.540 \\
\hline $1: \mathrm{G} \_7: \mathrm{H} 8$ & $1: \mathrm{G}_{-} 7: \mathrm{H} 1^{\prime}$ & 2.374 & 4.409 \\
\hline $1: \mathrm{G} \_7: \mathrm{H} 8$ & $1: \mathrm{G}_{-} 7: \mathrm{H} 2^{\prime}$ & 1.938 & 5.000 \\
\hline $1: \mathrm{G}^{-} 7: \mathrm{H} 1^{\prime}$ & $1: A_{-} 6: H 2$ & 2.462 & 4.572 \\
\hline $1: \mathrm{G}^{-} 7: \mathrm{H} 2^{\prime}$ & $1: \mathrm{G} \_8: \mathrm{H} 8$ & 1.800 & 3.028 \\
\hline $1: \mathrm{G}^{-} 7: \mathrm{H} 2^{\prime}$ & $1: \mathrm{G}_{-} 8: \mathrm{H} 1^{\prime}$ & 2.995 & 5.562 \\
\hline $1: \mathrm{G}^{-} 7: \mathrm{H} 3^{\prime}$ & $1: \mathrm{G}_{-} 7: \mathrm{H} 8$ & 1.800 & 5.000 \\
\hline $1: \mathrm{G}^{-} 7: \mathrm{H} 3^{\prime}$ & $1: \mathrm{G} \_8: \mathrm{H} 8$ & 1.931 & 5.000 \\
\hline $1: \mathrm{G} \_8: \mathrm{H} 8$ & $1: \mathrm{G}^{-} 7: \mathrm{H} 1^{\prime}$ & 2.675 & 4.968 \\
\hline $1: \mathrm{G} \_8: \mathrm{H} 8$ & $1: \mathrm{G}_{-} 8: \mathrm{H} 1^{\prime}$ & 2.818 & 5.233 \\
\hline $1: \mathrm{G} \_8: \mathrm{H} 8$ & $1: \mathrm{G}_{-} 8: \mathrm{H} 2^{\prime}$ & 3.057 & 5.676 \\
\hline $1: G \_8: H 8$ & $1: G_{-} 7: \mathrm{H} 8$ & 3.200 & 5.943 \\
\hline $1: G_{-} 8: \mathrm{H} 8$ & $1: \mathrm{C}_{-} 9: \mathrm{H} 5$ & 3.137 & 5.826 \\
\hline $1: \mathrm{G} \_8: \mathrm{H} 1{ }^{\prime}$ & $1: \mathrm{C}_{-} 9: \mathrm{H} 6$ & 3.149 & 5.849 \\
\hline $1: \mathrm{G}_{-} 8: \mathrm{H} 2{ }^{\prime}$ & $1: \mathrm{G}_{-} 8: \mathrm{H} 1^{\prime}$ & 1.874 & 3.480 \\
\hline $1: \mathrm{G} \_8: \mathrm{H} 2^{\prime}$ & $1: C_{-} 9: \mathrm{H} 5$ & 2.654 & 4.928 \\
\hline $1: \mathrm{G}^{-} 8: \mathrm{H} 2^{\prime}$ & $1: \mathrm{C}_{-} 9: \mathrm{H} 6$ & 1.800 & 3.104 \\
\hline $1: G \_8: H 3{ }^{\prime}$ & $1: \mathrm{G} \_8: \mathrm{H} 8$ & 1.973 & 3.664 \\
\hline $1: \mathrm{G}_{-}^{-} 8: \mathrm{H} 3^{\prime}$ & $1: \mathrm{G}^{-} 8: \mathrm{H} 1^{\prime}$ & 2.515 & 4.672 \\
\hline $1:$ G_8 :H3 ' & $1: C_{-} 9: \mathrm{H} 5$ & 2.525 & 4.689 \\
\hline $1: \mathrm{G}^{-} 8: \mathrm{H} 3{ }^{\prime}$ & $1: \mathrm{C}^{-} 9: \mathrm{H} 6$ & 2.149 & 3.990 \\
\hline $1: \mathrm{G} \_8: \mathrm{H} 4{ }^{\prime}$ & $1: \mathrm{G} \_8: \mathrm{H} 8$ & 2.583 & 5.000 \\
\hline $1: \mathrm{G}^{-} 8: \mathrm{H} 4{ }^{\prime}$ & $1: \mathrm{G}^{-} 8: \mathrm{H1}{ }^{\prime}$ & 2.645 & 4.911 \\
\hline $1: \mathrm{C}_{-} 9: \mathrm{H} 5$ & $1: \mathrm{U} 3{ }^{\prime} \_10: \mathrm{H} 5$ & 2.520 & 4.680 \\
\hline $1: \mathrm{C}^{-} 9: \mathrm{H} 6$ & $1: \mathrm{G} \_8: \mathrm{H} 8$ & 2.835 & 5.265 \\
\hline $1: \mathrm{C}_{2} 9: \mathrm{H} 6$ & $1: C_{2} 9: \mathrm{H} 5$ & 1.800 & 3.160 \\
\hline $1: \mathrm{C}_{-} 9: \mathrm{H} 6$ & $1: \mathrm{U} 3{ }^{\prime}{ }_{1} 10: \mathrm{H} 6$ & 2.910 & 5.404 \\
\hline $1: \mathrm{C}_{-} 9: \mathrm{H} 1{ }^{\prime}$ & $1: \mathrm{G} \_8: \mathrm{H} 2{ }^{\prime}$ & 2.875 & 5.339 \\
\hline $1: \mathrm{C}_{-} 9: \mathrm{H} 1{ }^{\prime}$ & $1: \mathrm{C}_{-} 9: \mathrm{H} 6$ & 2.545 & 4.727 \\
\hline $1: \mathrm{C} \_9: \mathrm{H} 1{ }^{\prime}$ & $1:$ C_9:H3 ' & 2.478 & 4.602 \\
\hline $1: \mathrm{C}_{-} 9: \mathrm{H} 2{ }^{\prime}$ & $1: C_{-} 9: \mathrm{H} 6$ & 2.394 & 5.000 \\
\hline $1: \mathrm{C}_{-} 9: \mathrm{H} 2{ }^{\prime}$ & $1: \mathrm{C}_{-} 9: \mathrm{H} 1{ }^{\prime}$ & 1.869 & 3.472 \\
\hline $1: \mathrm{C}_{-} 9: \mathrm{H} 3{ }^{\prime}$ & $1: \mathrm{C}_{-} 9: \mathrm{H} 6$ & 1.928 & 3.580 \\
\hline $1: \mathrm{C}_{-} 9: \mathrm{H} 5$ & $1: \mathrm{C}_{-} 9: \mathrm{H} 3{ }^{\prime}$ & 2.682 & 4.981 \\
\hline $1: \mathrm{C}_{-} 9: \mathrm{H} 4{ }^{\prime}$ & $1: \mathrm{C}_{-} 9: \mathrm{H} 1^{\prime}$ & 2.490 & 4.624 \\
\hline $1: \mathrm{U} 3{ }^{\prime} \_10: \mathrm{H} 5$ & $1: \mathrm{C}_{-} 9: \mathrm{H} 6$ & 3.010 & 5.590 \\
\hline $1: 3^{\prime}{ }^{\prime}-10:$ H5 & $1: \mathrm{C}_{-} 9: \mathrm{H} 2^{\prime}$ & 2.450 & 4.551 \\
\hline $1: \mathrm{U3}^{\prime}{ }^{\prime}{ }_{10}: \mathrm{H} 5$ & $1: \mathrm{C}_{-} 9: \mathrm{H} 3{ }^{\prime}$ & 2.452 & 5.000 \\
\hline $1: \mathrm{U3}{ }^{\prime}{ }^{\prime}{ }^{10}: \mathrm{H} 6$ & $1: \mathrm{C}_{-} 9: \mathrm{H1}{ }^{\prime}$ & 2.773 & 5.149 \\
\hline $1: \mathrm{U3}{ }^{\prime} \_10: \mathrm{H} 6$ & $1: \mathrm{C}_{-} 9: \mathrm{H} 2{ }^{\prime}$ & 1.800 & 3.327 \\
\hline $1: \mathrm{U3}{ }^{\prime} \_10: \mathrm{H} 6$ & $1: \mathrm{C}_{-} 9: \mathrm{H} 3{ }^{\prime}$ & 2.085 & 3.872 \\
\hline $1: \mathrm{U3}{ }^{\prime} \_10: \mathrm{H} 6$ & $1: \mathrm{U3}{ }^{\prime}{ }^{\prime} 10: \mathrm{H} 5$ & 1.800 & 3.267 \\
\hline $1: \mathrm{U}^{\prime}{ }^{\prime} \_10: \mathrm{H} 1^{\prime}$ & $1: \mathrm{U} 3{ }^{\prime} \_10: \mathrm{H} 6$ & 2.557 & 4.749 \\
\hline $1: \mathrm{U} 3{ }^{\prime}{ }^{\prime} 10: \mathrm{H} 1{ }^{\prime}$ & $1: \mathrm{C}_{-} 9: \mathrm{H} 2{ }^{\prime}$ & 2.642 & 4.907 \\
\hline
\end{tabular}




\begin{tabular}{|c|c|c|c|}
\hline $1: \mathrm{U}^{\prime}{ }^{\prime} \quad 10: \mathrm{H} 2^{\prime}$ & $1: \mathrm{U} 3{ }^{\prime} \_10: \mathrm{H} 6$ & 2.288 & 4.249 \\
\hline $1: \mathrm{U}^{\prime}{ }^{-}{ }^{-} 10: \mathrm{H} 2^{\prime}$ & $1: \mathrm{U} 3^{\prime}-10: \mathrm{H1}$ & 2.024 & 3.760 \\
\hline $1: \mathrm{U}^{\prime}{ }^{\prime}-10: \mathrm{H} 3{ }^{\prime}$ & $1: \mathrm{U} 3{ }^{\prime}-10: \mathrm{H} 6$ & 1.926 & 3.578 \\
\hline $1: \mathrm{U}^{\prime}{ }^{\prime}-10: \mathrm{H} 3{ }^{\prime}$ & $1: \mathrm{U}^{\prime}{ }^{-}-10: \mathrm{H1}$ & 2.279 & 4.233 \\
\hline $1: \mathrm{U}^{\prime}{ }^{\prime}-10: \mathrm{H} 4{ }^{\prime}$ & $1: \mathrm{U}^{\prime}{ }^{\prime}-10: \mathrm{H1}$ & 2.477 & 4.601 \\
\hline $1: \mathrm{U}^{\prime}{ }^{\prime}{ }^{-} 10: \mathrm{H} 4{ }^{\prime}$ & $1: \mathrm{U} 3{ }^{\prime}{ }^{-} 10: \mathrm{H} 6$ & 2.466 & 5.000 \\
\hline $1: 5^{\prime}-11: \mathrm{H} 8$ & $1: C \_1 \overline{2}: \mathrm{H} 6$ & 2.682 & 4.981 \\
\hline $1:$ G5 '-11:H8 & $1: G \overline{5}{ }^{\prime}{ }^{\prime} 11: \mathrm{H} 1^{\prime}$ & 2.596 & 4.821 \\
\hline 1:G5 '-11:H8 & $1: \mathrm{G}^{\prime}{ }^{-}-11: \mathrm{H} 2{ }^{\prime}$ & 2.498 & 4.639 \\
\hline $1: \mathrm{G} 5{ }^{\prime}-11: \mathrm{H} 8$ & $1: \mathrm{G}^{\prime}{ }^{\prime}-11: \mathrm{H} 3{ }^{\prime}$ & 2.225 & 4.132 \\
\hline $1: \mathrm{G}^{\prime}{ }^{-} 11: \mathrm{H} 1^{\prime}$ & $1: \mathrm{G}^{\prime}{ }^{-}-11: \mathrm{H} 3^{\prime}$ & 2.559 & 4.752 \\
\hline $1: \mathrm{G} 5{ }^{\prime}{ }^{-} 11: \mathrm{H} 1^{\prime}$ & $1: \mathrm{G}^{\prime}{ }^{-}-11: \mathrm{H} 4{ }^{\prime}$ & 2.415 & 4.485 \\
\hline $1: \mathrm{G}^{\prime}{ }^{-} 11: \mathrm{H} 1^{\prime}$ & $1: C \_1 \overline{2}: \mathrm{H} 6$ & 2.863 & 5.318 \\
\hline $1: \mathrm{G} 5{ }^{\prime}{ }^{-} 11: \mathrm{H} 2{ }^{\prime}$ & $1: \mathrm{G} \overline{5}{ }^{\prime} \_11: \mathrm{H} 1^{\prime}$ & 1.896 & 3.522 \\
\hline $1: \mathrm{G}^{\prime}{ }^{-} 11: \mathrm{H} 2^{\prime}$ & $1: C_{-} 1 \overline{2}:$ H5 & 2.480 & 4.605 \\
\hline $1: \mathrm{G}^{\prime}{ }^{-} 11: \mathrm{H} 2^{\prime}$ & $1: \mathrm{C}^{-} 12: \mathrm{H} 6$ & 1.800 & 3.220 \\
\hline $1: \mathrm{G}^{\prime}{ }^{-} 11: \mathrm{H} 3{ }^{\prime}$ & $1: \mathrm{C}^{-} 12: \mathrm{H} 6$ & 2.057 & 3.821 \\
\hline $1: \mathrm{G} 5 \mathrm{I}^{\prime}-11: \mathrm{H} 8$ & $1: G \overline{5}{ }^{\prime}{ }^{\prime} 11: \mathrm{H} 4{ }^{\prime}$ & 3.017 & 5.602 \\
\hline $1: \mathrm{G} 5 \mathrm{I}^{-}-11: \mathrm{H} 8$ & $1: \mathrm{C} 1 \overline{2}: \mathrm{H} 5$ & 3.603 & 6.000 \\
\hline $1: \mathrm{C} \_1 \overline{2}: \mathrm{H} 5$ & $1: \overline{5}^{\prime}{ }^{\prime} \quad 11: \mathrm{H} 3{ }^{\prime}$ & 2.775 & 5.153 \\
\hline $1: \mathrm{C}^{-} 12: \mathrm{H} 6$ & $1: \mathrm{C} \quad 1 \overline{2}: \mathrm{H} 5$ & 1.800 & 3.118 \\
\hline $1: \mathrm{C}^{-} 12: \mathrm{H} 6$ & $1: \mathrm{C}^{-} 12: \mathrm{H} 2^{\prime}$ & 2.173 & 5.000 \\
\hline $1: \mathrm{C}^{-} 12: \mathrm{H}^{\prime}$ & $1: \mathrm{G}^{\prime}{ }^{\prime} \quad 11: \mathrm{H} 2^{\prime}$ & 2.908 & 5.401 \\
\hline $1: \mathrm{C}^{-} 12: \mathrm{HI}^{\prime}$ & $1:$ C_1 $1 \overline{2}: \mathrm{H} 6$ & 2.482 & 4.610 \\
\hline $1: \mathrm{C}^{-} 12: \mathrm{H}^{\prime}$ & $1: \mathrm{C}^{-} 12: \mathrm{H} 2^{\prime}$ & 1.850 & 3.436 \\
\hline $1: \mathrm{C}^{-} 12: \mathrm{HI}^{\prime}$ & $1: \mathrm{C}^{-} 13: \mathrm{H} 6$ & 2.957 & 5.492 \\
\hline $1: \mathrm{C}^{-} 12: \mathrm{H} 2{ }^{\prime}$ & $1: \mathrm{C}^{-} 13: \mathrm{H} 5$ & 2.857 & 5.306 \\
\hline 1: C_12:H2' & $1: \mathrm{C}^{-} 13: \mathrm{H} 6$ & 1.800 & 3.296 \\
\hline $1: \mathrm{C}^{-} 12: \mathrm{H} 3{ }^{\prime}$ & $1: \mathrm{C}^{-} 12: \mathrm{H} 6$ & 1.935 & 3.593 \\
\hline $1: \mathrm{C}^{-} 12: \mathrm{H} 3{ }^{\prime}$ & $1: \mathrm{C}^{-} 12: \mathrm{H} 1^{\prime}$ & 2.134 & 3.963 \\
\hline $1: \mathrm{C}^{-} 12: \mathrm{H} 4{ }^{\prime}$ & $1: \mathrm{C}^{-} 12: \mathrm{H}^{\prime}{ }^{\prime}$ & 2.477 & 4.599 \\
\hline $1: \mathrm{C}^{-} 13: \mathrm{H} 6$ & $1: \mathrm{C}_{-} 13: \mathrm{H} 5$ & 1.800 & 3.323 \\
\hline $1: \mathrm{C}^{-} 13: \mathrm{H} 6$ & $1: \mathrm{C}_{-} 13: \mathrm{H}^{\prime}{ }^{\prime}$ & 2.011 & 3.735 \\
\hline $1: \mathrm{C}^{-} 13: \mathrm{H} 1^{\prime}$ & 1: C_12: ${ }^{-1} 2^{\prime}$ & 2.775 & 5.153 \\
\hline $1: \mathrm{C}^{-} 13: \mathrm{H}^{\prime}$ & $1: \mathrm{C}^{-} 13: \mathrm{H} 6$ & 2.588 & 4.807 \\
\hline $1: \mathrm{C}_{-}^{-} 13: \mathrm{H} 1^{\prime}$ & $1: \mathrm{C}_{-} 13: \mathrm{H} 3^{\prime}$ & 2.321 & 4.310 \\
\hline $1: \mathrm{C}_{-}^{-} 13: \mathrm{H} 1^{\prime}$ & $1: \mathrm{C}_{-}{ }^{-13}: \mathrm{H} 4{ }^{\prime}$ & 2.231 & 4.143 \\
\hline $1: \mathrm{C}_{-}^{-} 13: \mathrm{H} 1^{\prime}$ & $1: \mathrm{G}^{-} 14: \mathrm{H} 8$ & 3.288 & 6.000 \\
\hline $1: \mathrm{C}_{-}^{-} 13: \mathrm{H} 2{ }^{\prime}$ & $1: \mathrm{C}_{-} 13: \mathrm{H}^{\prime}{ }^{\prime}$ & 1.861 & 3.455 \\
\hline $1: \mathrm{G}^{-} 14: \mathrm{H} 8$ & $1: \mathrm{C}_{-} 13: \mathrm{H} 6$ & 2.968 & 5.512 \\
\hline $1: \mathrm{G}^{-} 14: \mathrm{H} 8$ & $1: \mathrm{C}^{-} 13: \mathrm{H} 2^{\prime}$ & 1.983 & 3.682 \\
\hline $1: \mathrm{G}^{-} 14: \mathrm{H} 8$ & $1: \mathrm{C}_{-} 13: \mathrm{H}^{\prime}{ }^{\prime}$ & 1.800 & 3.251 \\
\hline $1: \mathrm{G}^{-} 14: \mathrm{H} 8$ & $1: \mathrm{G}^{-} 14: \mathrm{H} 2^{\prime}$ & 2.022 & 5.000 \\
\hline $1: \mathrm{G}^{-} 14: \mathrm{H} 1^{\prime}$ & $1: \mathrm{G}^{-} 14: \mathrm{H} 8$ & 2.785 & 5.173 \\
\hline $1: \mathrm{G}_{-14} 14 \mathrm{H} 1^{\prime}$ & $1: \mathrm{G}^{-} 14: \mathrm{H} 2{ }^{\prime}$ & 1.993 & 3.701 \\
\hline $1: \mathrm{G}^{-} 14: \mathrm{HI}{ }^{\prime}$ & $1: \mathrm{G}^{-} 14: \mathrm{H}^{\prime}{ }^{\prime}$ & 2.818 & 5.233 \\
\hline $1: \mathrm{G}^{-14} 14: \mathrm{H} 3{ }^{\prime}$ & $1:$ G_14:H8 & 2.190 & 4.067 \\
\hline $1: \mathrm{G}^{-} 14: \mathrm{H} 4{ }^{\prime}$ & $1: \mathrm{G}^{-} 14: \mathrm{H} 1^{\prime}$ & 2.486 & 4.617 \\
\hline $1: \mathrm{A} \_16: \mathrm{H} 2$ & $1: \mathrm{G} \_17: \mathrm{H} 1^{\prime}$ & 2.400 & 4.457 \\
\hline $1: \mathrm{A}^{-} 16: \mathrm{H} 8$ & $1: \mathrm{A}^{-} 16: \mathrm{H} 2^{\prime}$ & 2.246 & 5.000 \\
\hline $1: \mathrm{A} \_16: \mathrm{H} 8$ & $1: \mathrm{G} \_17: \mathrm{H} 8$ & 2.766 & 5.136 \\
\hline $1: \mathrm{A}^{-} 16: \mathrm{H} 1^{\prime}$ & $1: \mathrm{A}^{-} 16: \mathrm{H} 8$ & 2.755 & 5.117 \\
\hline $1: \mathrm{A}_{-} 16: \mathrm{H} 1^{\prime}$ & $1: \mathrm{A} \_16: \mathrm{H} 2^{\prime}$ & 1.945 & 3.612 \\
\hline $1: \mathrm{A}^{-} 16: \mathrm{H} 1^{\prime}$ & $1: \mathrm{A}^{-} 16: \mathrm{H} 3^{\prime}$ & 2.612 & 4.851 \\
\hline $1: \mathrm{A}^{-} 16: \mathrm{H} 1^{\prime}$ & $1: \mathrm{A}^{-} 16: \mathrm{H} 4^{\prime}$ & 2.464 & 4.576 \\
\hline $1: \mathrm{A}^{-} 16: \mathrm{H} 2{ }^{\prime}$ & $1: \mathrm{G}^{-} 17: \mathrm{H} 8$ & 1.989 & 3.695 \\
\hline $1: \mathrm{A}^{-} 16: \mathrm{H}^{\prime}$ & $1: \mathrm{A}^{-} 16: \mathrm{H} 8$ & 2.129 & 3.953 \\
\hline
\end{tabular}




\begin{tabular}{|c|c|c|c|}
\hline $1:$ A_16:H3' & $1: G \_17: \mathrm{H} 8$ & 1.800 & 5.000 \\
\hline $1: \mathrm{G}^{-} 17: \mathrm{H} 3{ }^{\prime}$ & $1: \mathrm{G}^{-} 17: \mathrm{H} 8$ & 1.800 & 5.000 \\
\hline $1: \mathrm{G}^{-} 17: \mathrm{H} 8$ & $1: \mathrm{A}^{-} 16: \mathrm{H} 1^{\prime}$ & 3.552 & 6.000 \\
\hline $1: \mathrm{G}^{-} 17: \mathrm{H} 8$ & $1: \mathrm{C}_{-} 18: \mathrm{H} 5$ & 2.955 & 5.487 \\
\hline $1: \mathrm{G}^{-} 17: \mathrm{H} 1{ }^{\prime}$ & $1: \mathrm{G}^{-} 17: \mathrm{H} 8$ & 2.529 & 4.697 \\
\hline $1: \mathrm{G}^{-} 17: \mathrm{H} 2{ }^{\prime}$ & $1: \mathrm{G}^{-} 17: \mathrm{H} 8$ & 2.120 & 5.000 \\
\hline $1: \mathrm{G}^{-} 17: \mathrm{H} 2{ }^{\prime}$ & $1: \mathrm{C}_{-}^{-1} 18: \mathrm{H} 6$ & 1.800 & 3.194 \\
\hline $1: \mathrm{G}^{-} 17: \mathrm{H} 3{ }^{\prime}$ & $1: \mathrm{C}_{-} 18: \mathrm{H} 6$ & 1.945 & 3.612 \\
\hline $1: \mathrm{G}^{-} 17: \mathrm{H}^{\prime}{ }^{\prime}$ & $1: \mathrm{C}_{-}^{-18} 18: \mathrm{H} 5$ & 2.560 & 4.754 \\
\hline $1: \mathrm{C}^{-} 18: \mathrm{H} 5$ & $1: \mathrm{C}^{-} 18: \mathrm{H} 6$ & 1.800 & 3.299 \\
\hline $1: \mathrm{C}^{-} 18: \mathrm{H} 5$ & 1: $\mathrm{C}_{-} 18: \mathrm{H}^{\prime}{ }^{\prime}$ & 2.281 & 5.000 \\
\hline $1: \mathrm{C}^{-} 18: \mathrm{H} 6$ & $1: \mathrm{G}^{-} 17: \mathrm{H} 8$ & 2.835 & 5.265 \\
\hline $1: \mathrm{C}^{-} 18: \mathrm{H} 6$ & $1: \mathrm{G}^{-} 17: \mathrm{H} 1^{\prime}$ & 2.857 & 5.305 \\
\hline $1: \mathrm{C}^{-} 18: \mathrm{H} 6$ & $1: \mathrm{C}_{-} 18: \mathrm{H} 1^{\prime}$ & 2.522 & 4.683 \\
\hline $1: \mathrm{C}^{-} 18: \mathrm{H} 6$ & $1: \mathrm{C}_{-} 18: \mathrm{H} 2^{\prime}$ & 2.720 & 5.051 \\
\hline $1: \mathrm{C}^{-} 18: \mathrm{H} 6$ & $1: \mathrm{C}_{-} 18: \mathrm{H}^{\prime}{ }^{\prime}$ & 1.800 & 3.305 \\
\hline $1: \mathrm{C}^{-} 18: \mathrm{H} 1{ }^{\prime}$ & $1: \mathrm{C}_{-}-18: \mathrm{H} 2^{\prime}$ & 1.885 & 3.502 \\
\hline $1: \mathrm{C}^{-} 18: \mathrm{H} \mathbf{1}^{\prime}$ & $1: \mathrm{C}_{-} 18: \mathrm{H}^{\prime}{ }^{\prime}$ & 2.543 & 4.723 \\
\hline $1: \mathrm{C}^{-} 18: \mathrm{H} 1{ }^{\prime}$ & $1: \mathrm{C}_{-}-18: \mathrm{H}^{-}{ }^{\prime}$ & 2.240 & 4.160 \\
\hline $1: \mathrm{C}^{-} 18: \mathrm{H} 1{ }^{\prime}$ & $1: \mathrm{C}_{-}^{-1} 19: \mathrm{H} 6$ & 2.506 & 6.000 \\
\hline $1: \mathrm{C}^{-} 18: \mathrm{H}^{\prime}{ }^{\prime}$ & $1: \mathrm{C}_{-}^{-} 19: \mathrm{H} 6$ & 2.324 & 4.317 \\
\hline $1: \mathrm{G}^{-} 17: \mathrm{H} 2{ }^{\prime}$ & $1: \mathrm{C}_{-}^{-1} 18: \mathrm{H} 5$ & 2.396 & 4.449 \\
\hline $1: \mathrm{C}_{-}^{-} 19: \mathrm{H} 6$ & $1: \mathrm{C}_{-} 18: \mathrm{H} 2^{\prime}$ & 1.800 & 3.142 \\
\hline $1: \mathrm{C}^{-} 19: \mathrm{H} 6$ & $1: \mathrm{C}^{-} 19: \mathrm{H} 5$ & 1.800 & 3.177 \\
\hline $1: \mathrm{C}^{-} 19: \mathrm{H} 6$ & 1:C_-19:H2 ' & 2.304 & 5.000 \\
\hline $1: \mathrm{C}^{-} 19: \mathrm{H} 6$ & $1: \mathrm{C}^{-} 19: \mathrm{H}^{\prime}{ }^{\prime}$ & 1.800 & 3.309 \\
\hline $1: \mathrm{C}_{1} 19: \mathrm{H} 1{ }^{\prime}$ & $1: \mathrm{C}_{-} 18: \mathrm{H} 2^{\prime}$ & 2.875 & 5.339 \\
\hline $1: \mathrm{C}^{-} 19: \mathrm{H} 1{ }^{\prime}$ & $1: \mathrm{C}^{-} 19: \mathrm{H} 6$ & 2.598 & 4.825 \\
\hline $1: \mathrm{C}^{-} 19: \mathrm{H} 1{ }^{\prime}$ & 1:C_19:H2 ' & 1.815 & 3.371 \\
\hline $1: \mathrm{C}^{-} 19: \mathrm{H} \mathbf{1}^{\prime}$ & $1: \mathrm{C}^{-} 19: \mathrm{H}^{\prime}{ }^{\prime}$ & 2.586 & 4.803 \\
\hline $1: \mathrm{C} \_19: \mathrm{H} 1{ }^{\prime}$ & $1: \mathrm{P} 3{ }^{\prime} \_20: \mathrm{H} 8$ & 3.338 & 6.000 \\
\hline $1: \mathrm{C}^{-} 19: \mathrm{H} 2{ }^{\prime}$ & $1:$ P3 ' $20: \mathrm{H} 8$ & 1.800 & 3.198 \\
\hline $1: \mathrm{C}^{-1} 19: \mathrm{H} 2{ }^{\prime}$ & $1: \mathrm{P} 3{ }^{\prime} \_20: \mathrm{H} 1{ }^{\prime}$ & 2.533 & 4.704 \\
\hline $1: \mathrm{C}^{-} 19: \mathrm{H}^{\prime}{ }^{\prime}$ & $1: \mathrm{P} 3^{\prime}-20: \mathrm{H} 8$ & 2.049 & 3.805 \\
\hline $1: \mathrm{C}^{-19} 1 \mathrm{H} 4{ }^{\prime}$ & $1: \mathrm{C} \_1 \overline{9}: \mathrm{H} 1^{\prime}$ & 2.490 & 4.625 \\
\hline $1: \mathrm{P} \overline{3}{ }^{\prime} \_20: \mathrm{H} 2$ & $1: \mathrm{G} \overline{5}{ }^{\prime} \_1: \mathrm{H} 1^{\prime}$ & 3.149 & 5.849 \\
\hline $1:$ : $3{ }^{\prime}-20:$ H8 & $1:$ C_1 $19: \mathrm{H} 6$ & 3.270 & 6.000 \\
\hline $1:$ : $3{ }^{\prime}-20:$ H8 & $1: \mathrm{P} \overline{3}{ }^{\prime} \_20: \mathrm{H} 1^{\prime}$ & 2.832 & 5.259 \\
\hline $1:$ : $3{ }^{\prime} \_20: \mathrm{H} 8$ & $1:$ P3 ' $20: \mathrm{H} 2^{\prime}$ & 2.408 & 4.472 \\
\hline $1:$ :3 ' $20: \mathrm{H} 1{ }^{\prime}$ & $1: \mathrm{P} 3^{\prime} \_20: \mathrm{H} 2^{\prime}$ & 2.015 & 3.742 \\
\hline $1:$ :3 ' ${ }^{-} 20: \mathrm{H}{ }^{\prime}$ & $1:$ : $3{ }^{\prime}-20: \mathrm{H} 3{ }^{\prime}$ & 2.612 & 4.851 \\
\hline $1:$ : $3{ }^{\prime}{ }^{-} 20: \mathrm{H} 3{ }^{\prime}$ & $1: \mathrm{P} 3^{\prime}{ }^{-}-20: \mathrm{H} 8$ & 1.976 & 3.670 \\
\hline $1:$ P3 '_2 $0: \mathrm{H} 4{ }^{\prime}$ & $1:$ P3 '_20:H8 & 2.560 & 4.754 \\
\hline $1:$ P3 '_-20:H4 ' & $1:$ : 3 ' $20: \mathrm{H} 1{ }^{\prime}$ & 2.396 & 4.450 \\
\hline
\end{tabular}

\footnotetext{
a For the structure modeling of A6/A15/A16 only; ${ }^{b}$ For the structure modeling of A6/A5/A16 only; ${ }^{C}$ Loosened upper bound to $4.50 \AA$ for the structural modeling of A6/A5/A16.
} 


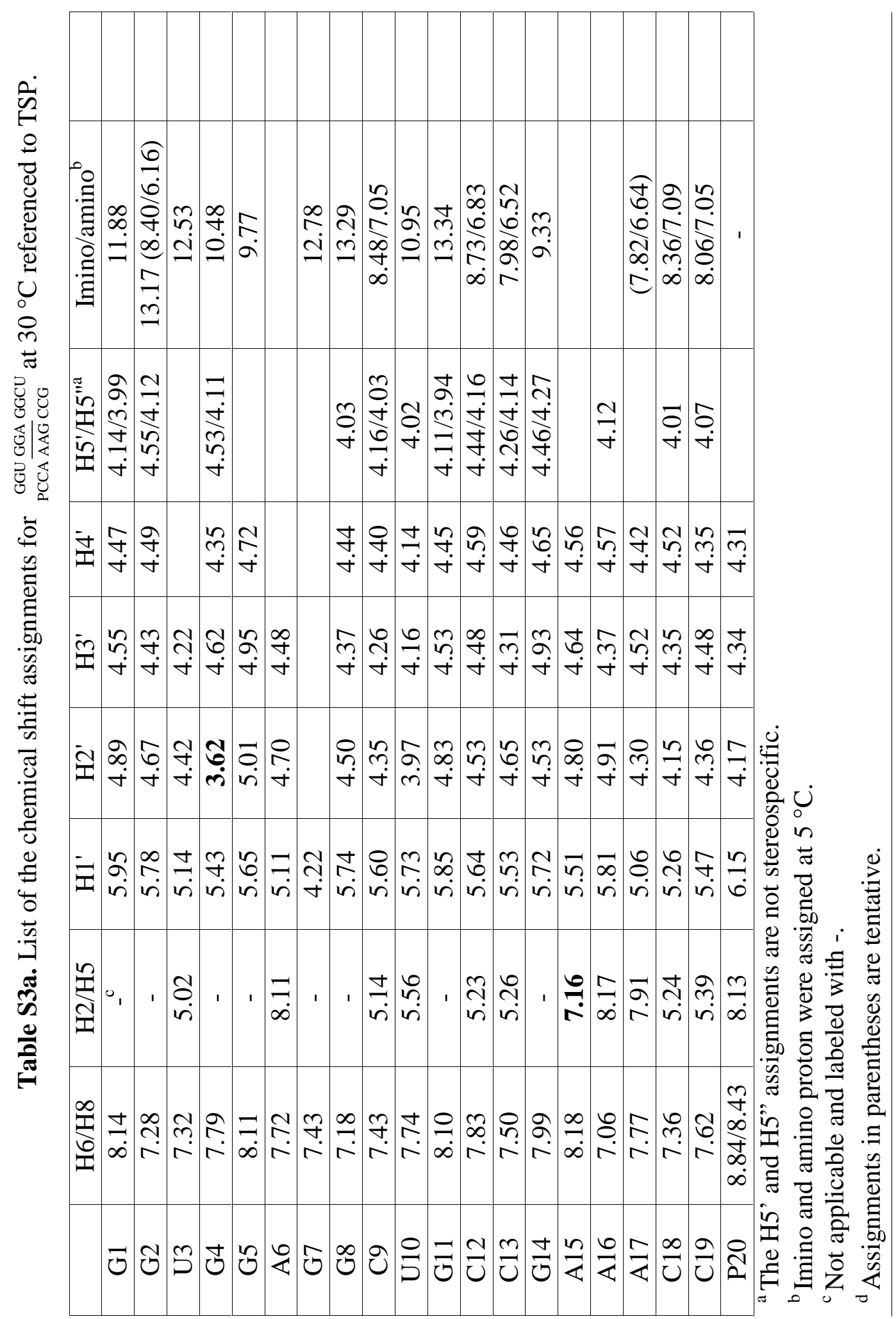




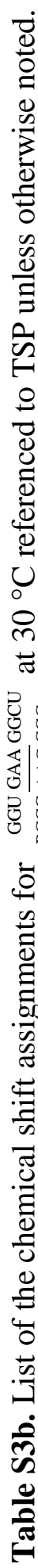

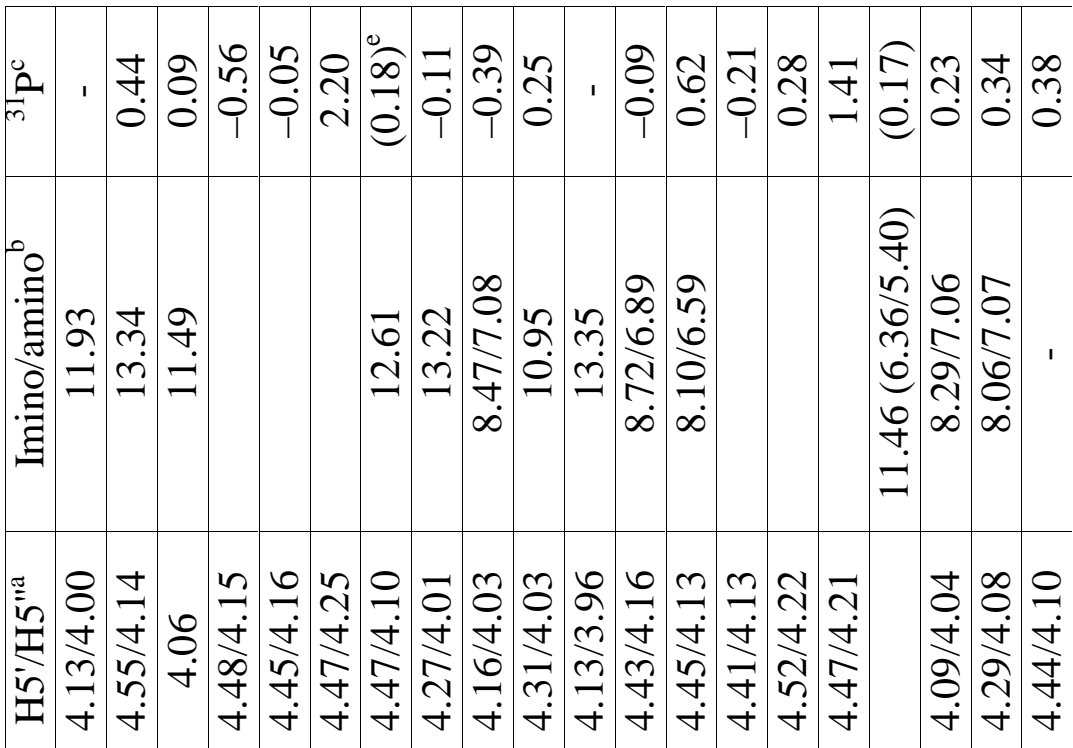

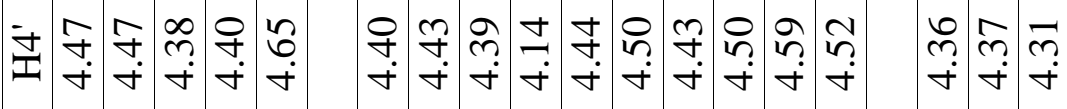

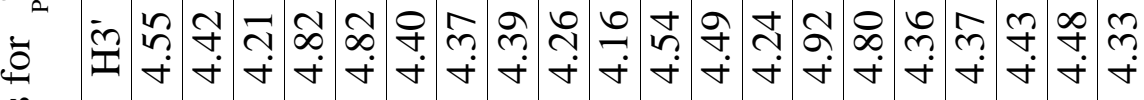

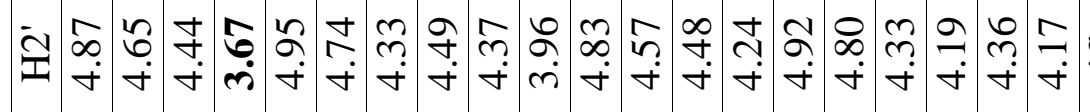

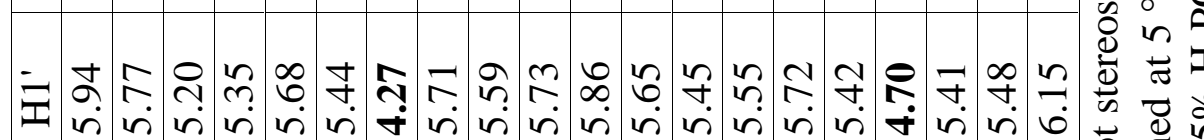

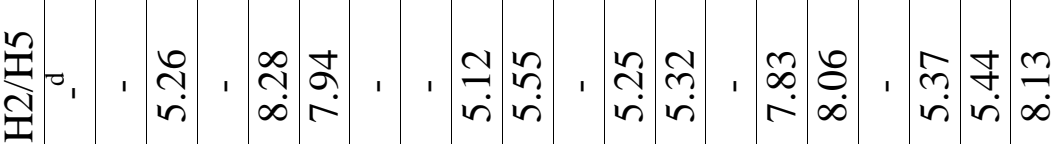

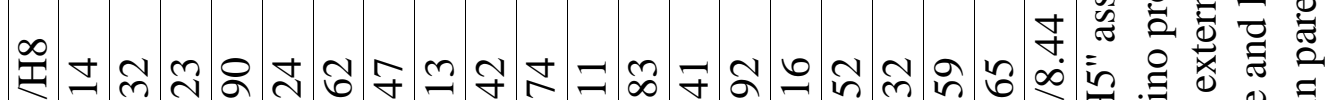

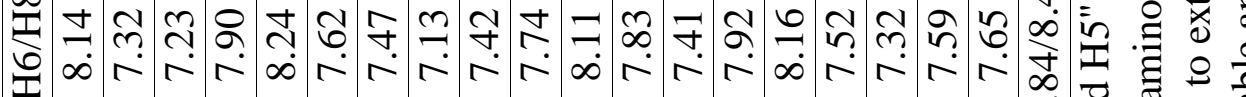

๕ุุ̊

O 
Figure S1. The $40 \mathrm{~ms}$ mixing time TOCSY spectra (H1'-sugar region) of $\begin{gathered}\text { GGU GGA GGCU } \\ \text { AAAG }_{\text {CCG }}\end{gathered}$

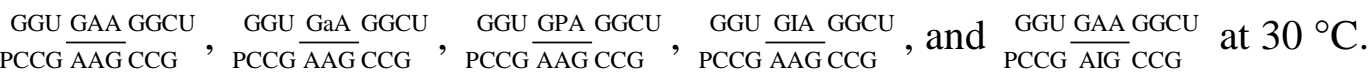
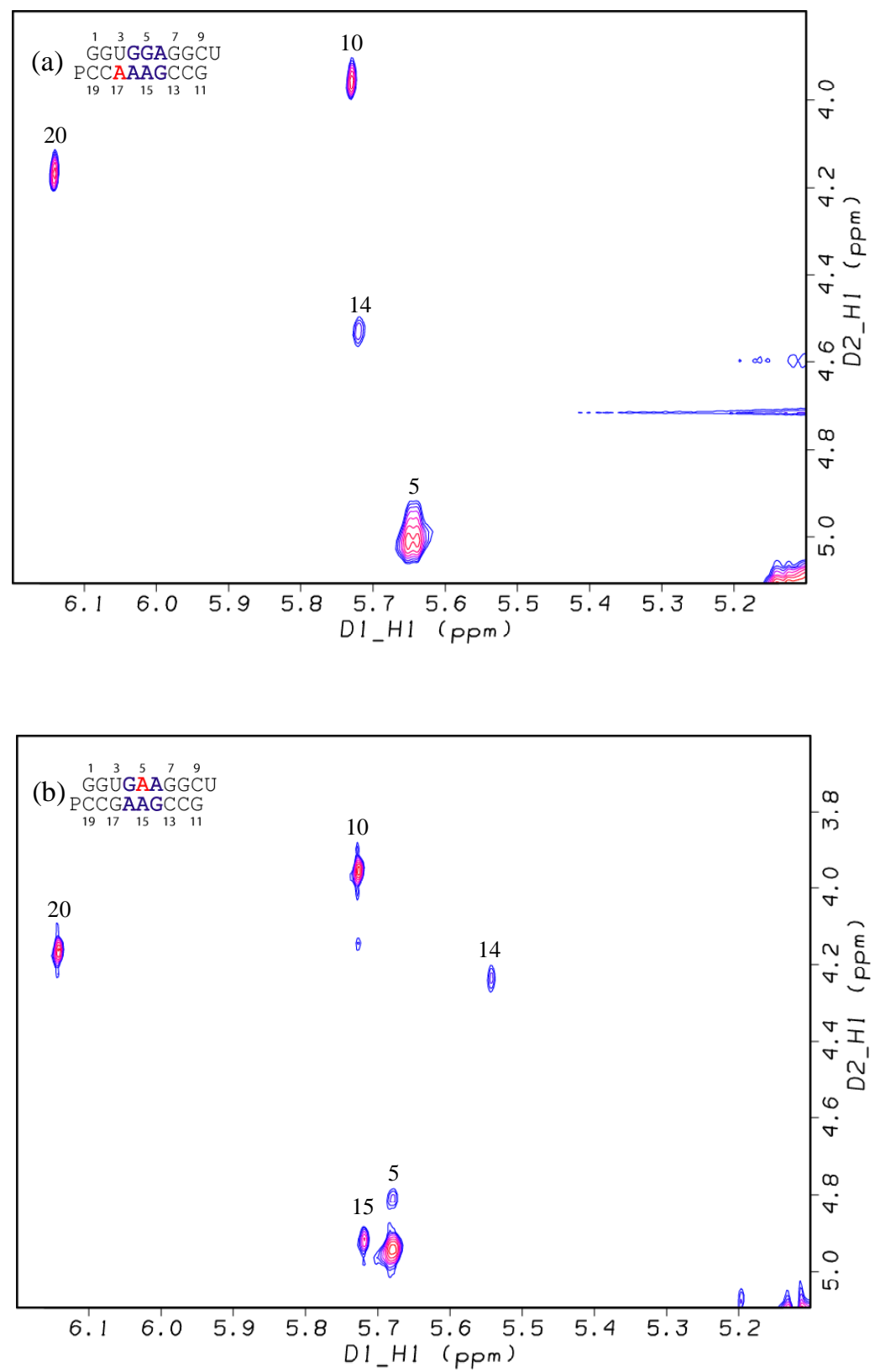

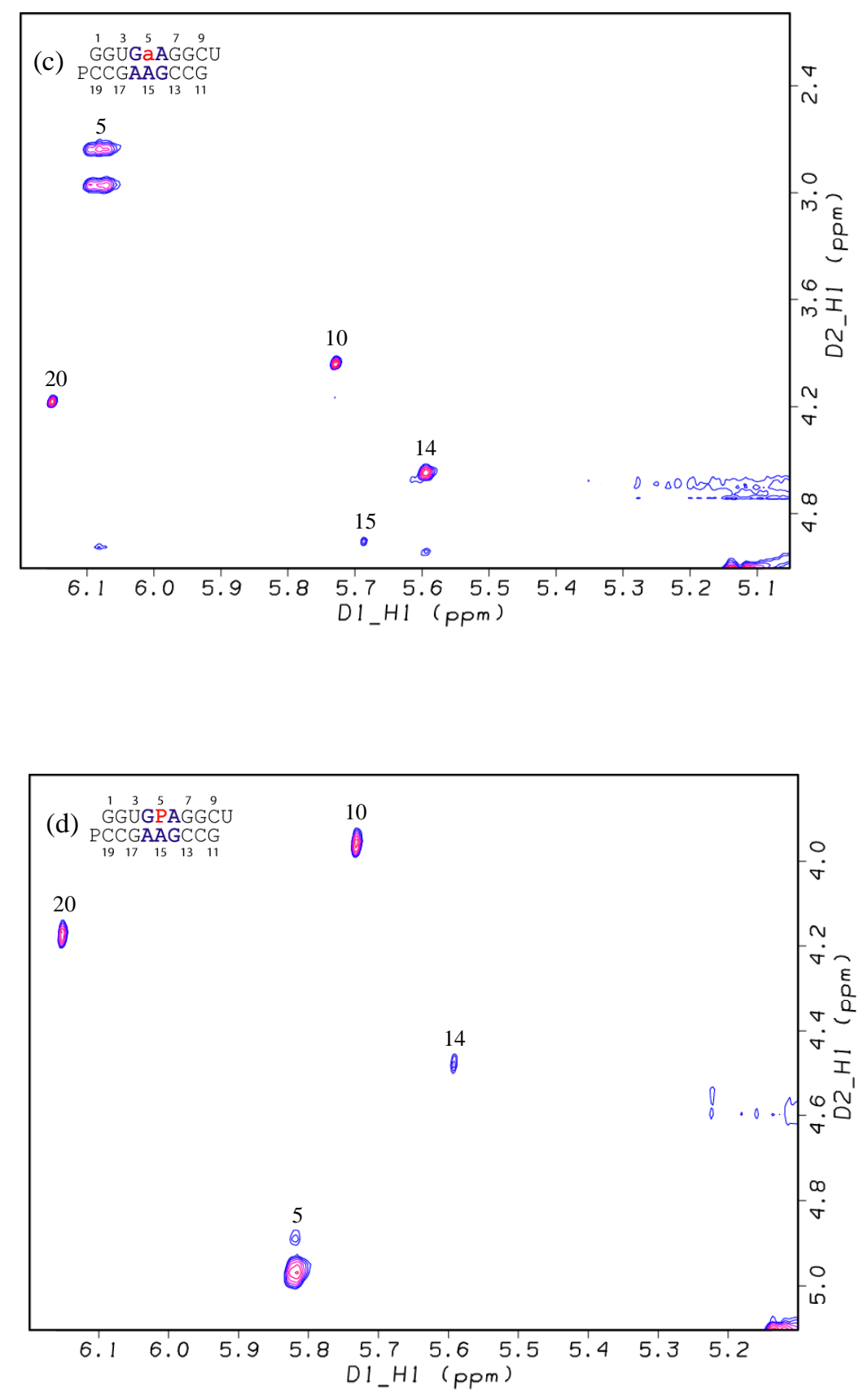

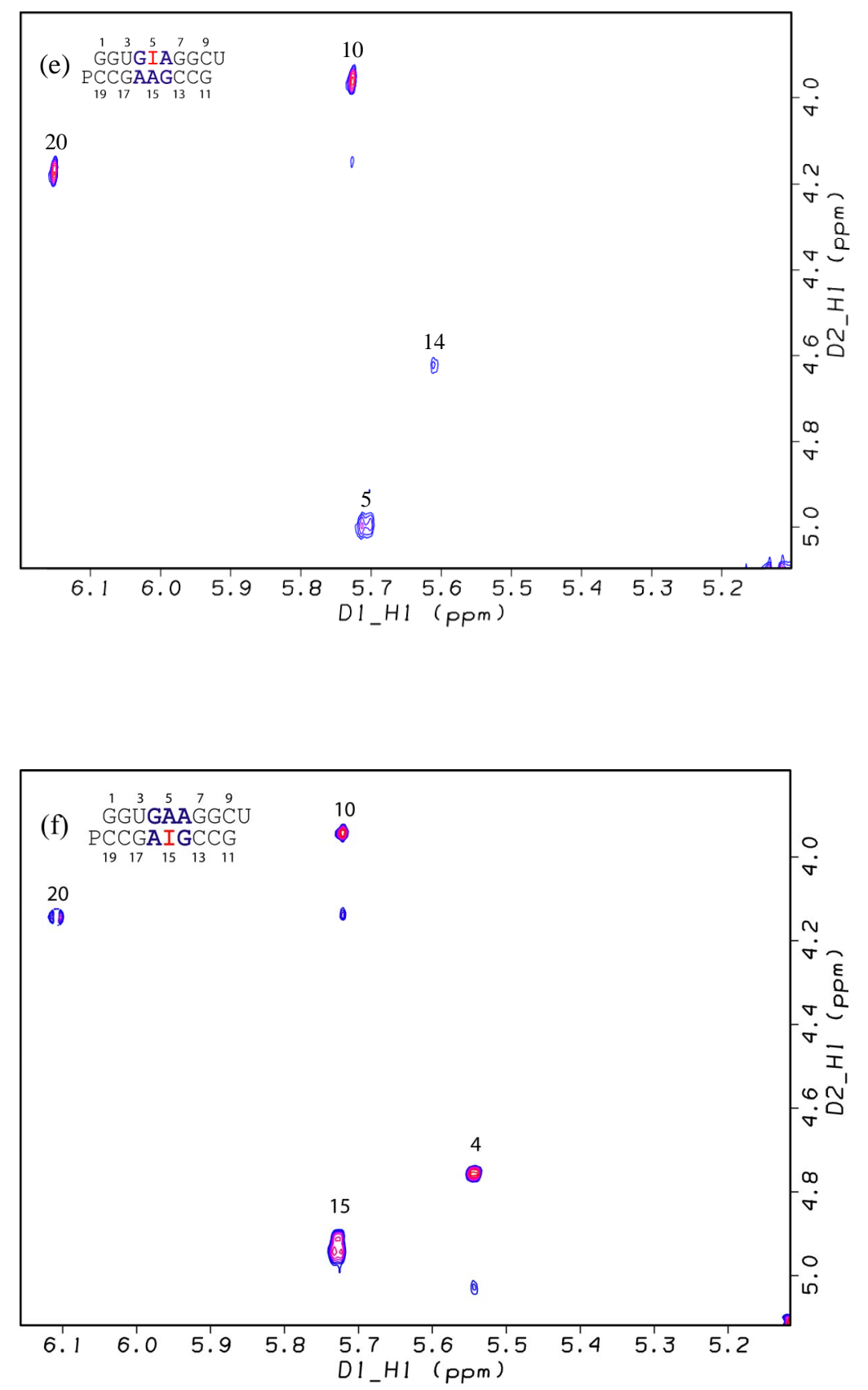
Figure S2. One-dimensional proton spectra (9-14.5 ppm) in 90:10 (v:v) $\mathrm{H}_{2} \mathrm{O}: \mathrm{D}_{2} \mathrm{O}$ at 5

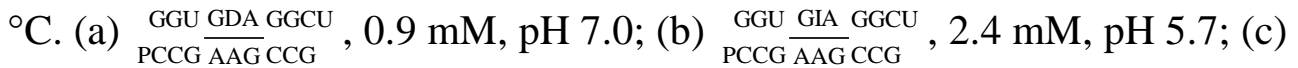

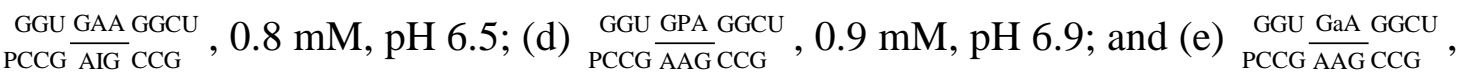
$1.6 \mathrm{mM}, \mathrm{pH}$ 6.9. Numbers on spectra correspond to assignments with numbering starting at left most (5') nucleotide of top strand and ending at left most (3') nucleotide of bottom strand. The value to the right of each duplex is the free energy increment in $\mathrm{kcal} / \mathrm{mol}$ for formation of the internal loop at $37^{\circ} \mathrm{C}$, at $\mathrm{pH} 7$ in $1 \mathrm{M} \mathrm{NaCl}$. Spectra for ${ }_{\text {PCCA }}^{\mathrm{GGAG}} \underset{\mathrm{AACG}}{\mathrm{GGC}}$ and PCCG $\frac{\text { GAA GGCU }}{\text { AAG }}$ CCG were published in ref (2).

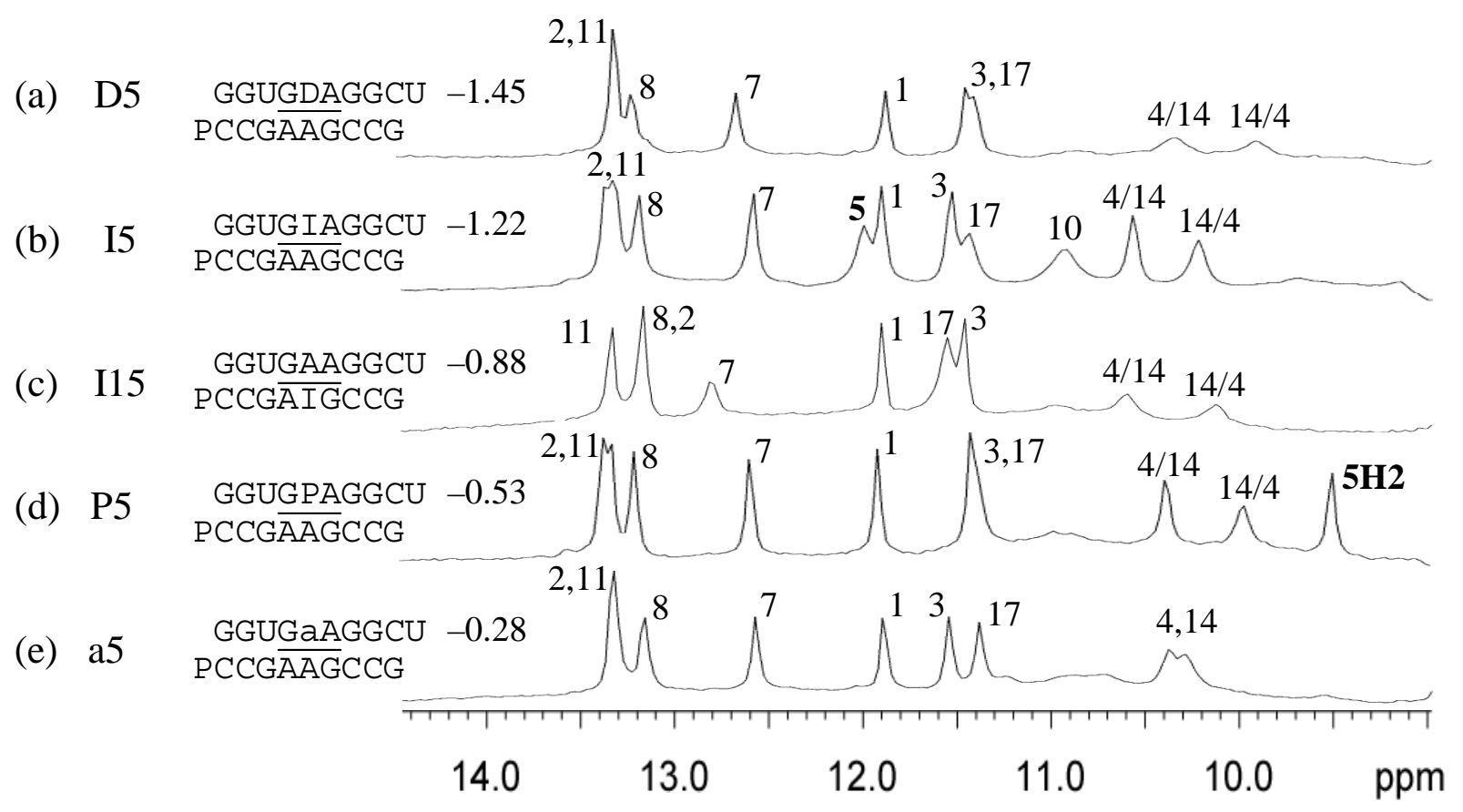


Figure S3:(H8/H6/H2)-(H1'/H5) region of the $400 \mathrm{~ms}$ mixing time NOESY spectrum of A5 duplex (Figure 2) at $30{ }^{\circ} \mathrm{C}$ in $1 \mathrm{M} \mathrm{NaCl}, 10 \mathrm{mM}$ sodium phosphate, $0.5 \mathrm{mM}$ disodium EDTA, pH7. As in Figure $3 \mathrm{~b}$ for the A5 sequence at $80 \mathrm{mM} \mathrm{NaCl}$, yellow and green circles connected by gray lines identify related cross peaks of major and minor conformations, respectively. Note that chemical shifts are only mildly salt dependent, although $\mathrm{A} 6 \mathrm{H} 1^{\prime}$ and $\mathrm{A} 16 \mathrm{H} 1^{\prime}$ overlap at $1 \mathrm{M} \mathrm{NaCl}$ so their cross peaks to $\mathrm{A} 15 \mathrm{H} 2$ (major conformation) and $\mathrm{A} 5 \mathrm{H} 2$ (minor conformation) are not resolved. $\mathrm{A} 15 \mathrm{H} 2$ and $\mathrm{G} 4 \mathrm{H} 8$ are also overlapped and the cross peak A15H2-G4H1' is overlapped with G4H8-G4H1'.

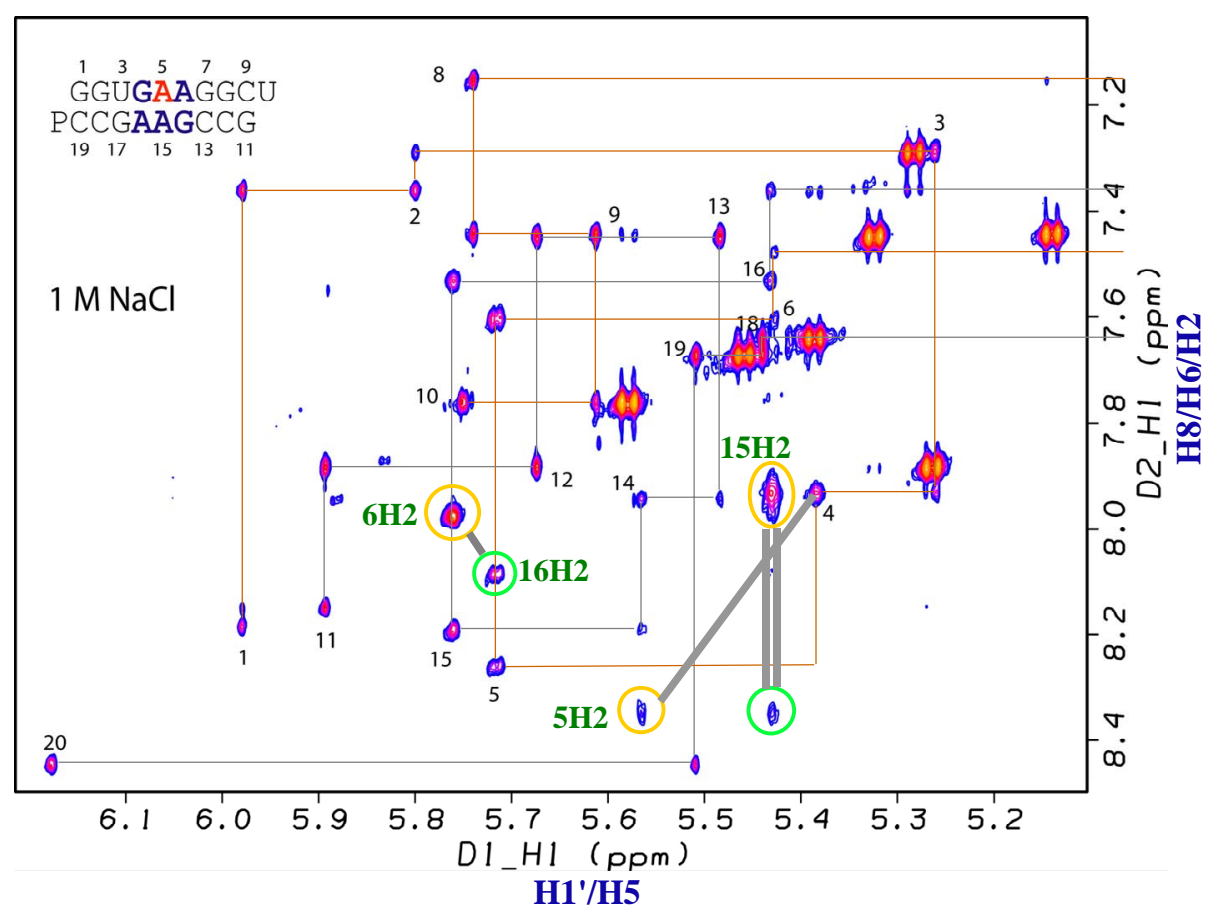

\section{References:}

1. Harms, J., Schluenzen, F., Zarivach, R., Bashan, A., Gat, S., Agmon, I., Bartels, H., Franceschi, F., and Yonath, A. (2001) High resolution structure of the large ribosomal subunit from a mesophilic Eubacterium. Cell 107, 679-688.

2. Chen, G., and Turner, D. H. (2006) Consecutive GA pairs stabilize medium size RNA internal loops. Biochemistry In press. 